\title{
A CLASSIFICATION OF MINKOWSKI PLANES OVER HALF-ORDERED FIELDS
}

\author{
GÜNTER F. STEINKE \\ Department of Mathematics \& Statistics \\ University of Canterbury \\ Private Bag 4800 \\ Christchurch, New Zealand
}

No. 171

February, 1999

\begin{abstract}
This paper concerns a construction of Minkowski planes over half-ordered fields [5] and [20]. Solving various functional equations the Klein-Kroll types of these Minkowski planes are determined with respect to $G$ - and $q$-translations and $(p, q)$-homotheties. Examples for some of the resulting types are given.
\end{abstract}




\title{
A CLASSIFICATION OF MINKOWSKI PLANES OVER HALF-ORDERED FIELDS
}

\author{
GüNTER F. STEINKE \\ Department of Mathematics \\ University of Canterbury \\ Private Bag 4800 \\ Christchurch, New Zealand \\ e-mail: G.Steinke@math.canterbury.ac.nz
}

\begin{abstract}
This paper concerns a construction of Minkowski planes over half-ordered fields [5] and [20]. Solving various functional equations the Klein-Kroll types of these Minkowski planes are determined with respect to $G$ - and $q$-translations and $(p, q)$ homotheties. Examples for some of the resulting types are given.
\end{abstract}

\section{InTRODUCTION AND Notation}

A Minkowski plane $\mathcal{M}=\left(P, \mathcal{K},\left\{\left\|_{+},\right\|_{-}\right\}\right)$consists of a set of points $P$, a set of at least two circles $\mathcal{K}$ (considered as subsets of $P$ ) and two equivalence relations $\|_{+}$ and $\|_{-}$on $P$ (parallelisms) such that three mutually non-parallel points (that is, neither $(+)$-parallel nor $(-)$-parallel) can be joined by a unique circle, such that the circles which touch a fixed circle $K$ at $p \in K$ partition $P \backslash|p|$ (where $|p|=|p|_{+} U|p|_{-}$ denotes the union of the two parallel classes of $p$ ), such that each parallel class meets each circle in a unique point (parallel projection), such that each $(+)$-parallel class and each $(-)$-parallel class intersect in a unique point, and such that there is a circle that contains at least three points (compare [18]).

Associated with every point $p$ of $\mathcal{M}$ there is an incidence structure, called the derived affine plane or residual plane $\mathcal{A}_{p}=\left(A_{p}, \mathcal{L}_{p}\right)$ at $p$, whose point set $A_{p}$ consists of all points of $\mathcal{M}$ that are not parallel to $p$ and whose set of lines $\mathcal{L}_{p}$ consists of all restrictions to $A_{p}$ of circles of $\mathcal{M}$ passing through $p$ and of all parallel classes not passing through $p$. Indeed, $\mathcal{M}$ is a Minkowski plane if and only if all incidence structures $\mathcal{A}_{p}$ are affine planes.

Let $\mathbb{F}$ be a coordinatizing ternary field of a derived affine plane of a Minkowski plane $\mathcal{M}$. Then $\mathcal{M}$ can be described as follows. The point set is $\overline{\mathbb{F}} \times \overline{\mathbb{F}}$, where $\overline{\mathbb{F}}=\mathbb{F} \cup\{\infty\}$.

1991 Mathematics Subject Classification. 51B20. 
Two points $(x, y)$ and $\left(x^{\prime}, y^{\prime}\right)$ are (+)- or (-)-parallel if and only if $x=x^{\prime}$ or $y=y^{\prime}$ respectively. Each circle $K$ of $\mathcal{M}$ is described by a function $f_{K}: \overline{\mathbb{F}} \rightarrow \overline{\mathbb{F}}$ as

$$
K=\left\{\left(x, f_{K}(x)\right) \mid x \in \overline{\mathbb{F}}\right\}
$$

We always use this representation for the Minkowski planes $\mathcal{M}(\mathbb{F} ; f, g)$ we define below. The axiom of parallel projection shows that each function $f_{K}$ is a permutation of $\overline{\mathbb{F}}$. The axiom of joining implies that the collection of all those permutations $f_{K}$ is a sharply 3 -transitive set of permutations of $\overline{\mathbb{F}}$. Conversely, each such incidence structure constructed from a sharply 3 -transitive set of permutations of $\overline{\mathbb{F}}$ is equivalent to a more general hyperbola structure or $\left(\mathrm{B}^{*}\right)$-geometry, that is, all axioms of a Minkowski plane are satisfied except the axiom of touching.

A half- (or pseudo-) ordered field $\mathbb{F}$ is a field with a multiplicative subgroup $\mathbb{P}$ of index two. In particular, $\mathbb{P}$ contains all non-zero squares of $\mathbb{F}$ so that a finite halfordered field cannot have characteristic two. Elements of $\mathbb{P}$ and of the other coset of non-zero elements are called positive and negative respectively. We write $x>0$ for $x \in \mathbb{P}$ and $x<0$, if $x$ is negative.

In [20] the notion of order-preserving, order-reversing and monotonic permutations of a half-ordered field $\mathbb{F}$ to permutations of $\overline{\mathbb{F}}$ as follows. Let

$$
\varepsilon\left(x_{1}, x_{2}, x_{3}\right)= \begin{cases}\left(x_{1}-x_{2}\right)\left(x_{2}-x_{3}\right)\left(x_{3}-x_{1}\right), & \text { if } x_{1}, x_{2}, x_{3} \neq \infty \\ x_{3}-x_{2}, & \text { if } x_{1}=\infty \\ x_{1}-x_{3}, & \text { if } x_{2}=\infty \\ x_{2}-x_{1}, & \text { if } x_{3}=\infty\end{cases}
$$

for mutually disctinct $x_{1}, x_{2}, x_{3} \in \overline{\mathbb{F}}$. We then say that a permutation $f$ of $\overline{\mathbb{F}}$ is orderpreserving or order-reversing if and only if $\varepsilon\left(f\left(x_{1}\right), f\left(x_{2}\right), f\left(x_{3}\right)\right) / \varepsilon\left(x_{1}, x_{2}, x_{3}\right)>0$ or $\varepsilon\left(f\left(x_{1}\right), f\left(x_{2}\right), f\left(x_{3}\right)\right) / \varepsilon\left(x_{1}, x_{2}, x_{3}\right)<0$, respectively, for all mutually disctinct $x_{1}, x_{2}, x_{3} \in \overline{\mathbb{F}}$. We call $f$ monotonic if $f$ is order-preserving or order-reversing. When $x_{3}=\infty$ and $f$ fixes that point one obtains the familiar definition of an order-preserving or order-reversing permutation of $\mathbb{F}$; cf. [4]. In the respective cases $(f(x)-f(y)) /(x-y)>0$ or $(f(x)-f(y)) /(x-y)<0$ for all distinct $x, y \in \mathbb{F}$.

We define $\Pi^{+}(\overline{\mathbb{F}})$ and $\Pi^{-}(\overline{\mathbb{F}})$ to be the collection of all order-preserving and all order-reversing permutations of $\overline{\mathbb{F}}$ respectively. Finally let $\Pi(\overline{\mathbb{F}})=\Pi^{+}(\overline{\mathbb{F}}) \cup \Pi^{-}(\overline{\mathbb{F}})$ be the collection of all monotonic permutations of $\overline{\mathbb{F}}$.

One readily verifies that $\Pi(\overline{\mathbb{F}})$ and $\Pi^{+}(\overline{\mathbb{F}})$ are groups with respect to composition of permutations. $\Pi^{+}(\overline{\mathbb{F}})$ is a normal subgroup of $\Pi(\overline{\mathbb{F}})$ of index 2 , and $\Pi^{-}(\overline{\mathbb{F}})$ is the other coset of $\Pi^{+}(\overline{\mathbb{F}})$ in $\Pi(\overline{\mathbb{F}})$.

We denote the projective linear group over the field $\mathbb{F}$ by $\operatorname{PGL}(2, \mathbb{F})$, that is, the quotient group formed by the general linear group $\mathrm{GL}(2, \mathbb{F})$ of $2 \times 2$ matrices modulo the non-zero scalar matrices. Unless we refer to a particular field we shall write $\operatorname{PGL}(2, \mathbb{F})$ as $\mathrm{PGL}_{2}$, that is, $\mathrm{PGL}_{2}$ and likewise the other groups are relative to the general half-ordered field $\mathbb{F}$. As ususal we identify an element of $\mathrm{PGL}_{2}$ represented 
by the $2 \times 2$ matrix $\left(\begin{array}{ll}a & b \\ c & d\end{array}\right), \operatorname{det}\left(\begin{array}{ll}a & b \\ c & d\end{array}\right)=a d-b c \neq 0$, with the fractional linear mapping $x \mapsto(a x+b) /(c x+d)$ operating in the standard way on $\bar{F}=\mathbb{F} \cup\{\infty\}$. We define

$$
\mathrm{PGL}_{2}^{+}=\mathrm{PGL}^{+}(2, \mathbb{F})=\Pi^{+}(\overline{\mathbb{F}}) \cap \operatorname{PGL}(2, \mathbb{F})
$$

this is a normal subgroup of index 2 in $\mathrm{PGL}_{2}$. Similarly, let

$$
\mathrm{PGL}_{2}^{-}=\mathrm{PGL}^{-}(2, \mathbb{F})=\Pi^{-}(\overline{\mathbb{F}}) \cap \operatorname{PGL}(2, \mathbb{F}) \text {. }
$$

An easy calculation shows that a permutation $\gamma: x \mapsto(a x+b) /(c x+d)$ belongs to $\mathrm{PGL}_{2}^{+}$or $\mathrm{PGL}_{2}^{-}$if and only if $a d-b c>0$ and $a d-b c<0$ respectively. Note that if one chooses a different representing matrix the determinant multiplies by a square in $\mathbb{F}$ which is positive; hence the above condition is independent of the representing matrix. It is well known that $\mathrm{PGL}_{2}$ is a sharply 3-transitive permutation group of $\overline{\mathbb{F}}$.

Let $f, g \in \Pi^{+}(\overline{\mathbb{F}})$. In $[20]$ we defined

$$
\mathcal{K}_{f, g}=\mathrm{PGL}_{2}^{+} \cup g^{-1} \mathrm{PGL}_{2}^{-} f \subseteq \Pi(\overline{\mathbb{F}}) .
$$

Then $\mathcal{M}(\mathbb{F} ; f, g)$ is the incidence structure whose circle set is $\mathcal{K}_{f, g}$, that is, each circle is the graph of a permutation in $\mathcal{K}_{f, g}$. We call $\mathrm{PGL}_{2}^{+}$and $g^{-1} \mathrm{PGL}_{2}^{-} f$ the positve and negative component of $\mathcal{K}_{f, g}$, respectively. (For $\mathbb{F}=\mathbb{R}, \mathrm{PGL}_{2}^{+}=\operatorname{PSL}(2, \mathbb{R})$ is the connected component of the identity of the topological group $\operatorname{PGL}(2, \mathbb{R})$.)

We say that two permutations $f, g \in \Pi^{+}(\overline{\mathbb{F}})$ have the fixed point property (FP) if and only if

$$
|\mathrm{Fix}(\gamma)| \neq 1 \text { for all } \gamma \in \mathrm{PGL}_{2}^{+} g^{-1} \mathrm{PGL}_{2}^{-} f
$$

where $\operatorname{Fix}(\gamma)$ is the collection of all points fixed by $\gamma$.

Then $\mathcal{M}(\mathbb{F} ; f, g)$ is a Minkowski plane if and only if $f$ and $g$ have the fixed point property (FP); see [20, Theorem 2.7]. Furthermore, $\mathcal{M}(\mathbb{F} ; f, g)$ is Miquelian, that is, each circle is the graph of a fractional linear mapping $x \mapsto(a x+b) /(c x+d)$, if and only if $f, g \in \mathrm{PGL}_{2}^{+} \alpha$ where $\alpha$ is an order-preserving automorphism of $\mathbb{F}$. Minkowski planes of this kind over $\mathbb{R}$ with the Euclidean ordering and their automorphism groups have been studied in [17], see also section 6 for examples.

I wish to thank H.-J. Kroll for his comments and suggestions.

\section{Automorphisms of $\mathcal{M}(\mathbb{F} ; f, g)$}

We always make the assumption that $f, g \in \Pi^{+}(\overline{\mathbb{F}})$ and that $f$ and $g$ satisfy the fixed point property (FP). There are four fundamental types of isomorphisms between such Minkowski planes, cf. [20]. Wé list the three types of isomorphisms we shall use later on. 
2.1. Isomorphisms induced by linear fractional maps:

$$
(x, y) \mapsto(\alpha(x), \beta(y))
$$

where $\alpha, \beta \in \mathrm{PGL}_{2}^{+}$. A circle $K_{\tau}, \tau \in \mathcal{F}_{f, g}$, is mapped to $K_{\beta \tau \alpha^{-1}}$. This map yields an isomorphism from $\mathcal{M}(\mathbb{F} ; f, g)$ to $\mathcal{M}\left(\mathbb{F} ; f \alpha^{-1}, g \beta^{-1}\right)$.

2.2. An isomorphism that maps (+)-parallel classes to $(-)$-parallel classes and $(-)$-parallel classes to $(+)$-parallel classes:

$$
(x, y) \mapsto(y, x)
$$

A circle $K_{\tau}, \tau \in \mathcal{F}_{f, g}$, is mapped to $K_{\tau^{-1}}$. This map yields an isomorphism from $\mathcal{M}(\mathbb{F} ; f, g)$ to $\mathcal{M}(\mathbb{F} ; g, f)$.

2.3. An isomorphism that maps the positive component of the circle set to the negative component and the negative component to the positive component:

$$
(x, y) \mapsto(f(x), \gamma g(y))
$$

where $\gamma \in \mathrm{PGL}_{2}^{-}$. A circle $K_{\tau}, \tau \in \mathcal{F}_{f, g}$, is mapped to $K_{\gamma g \tau f^{-1}}$. This map yields an isomorphism from $\mathcal{M}(\mathbb{F} ; f, g)$ to $\mathcal{M}\left(\mathbb{F} ; f^{-1}, \gamma g^{-1} \gamma^{-1}\right)$.

2.4. Let $\operatorname{Aut}(\mathbb{F})$ be the collection of all automorphisms of the field $\mathbb{F}$ and let

$$
\mathrm{P}_{2}=\operatorname{P\Gamma L}(2, \mathbb{F})=\mathrm{PGL}_{2} \operatorname{Aut}(\mathbb{F})
$$

be the collection of all semi-linear fractional permutations of $\overline{\mathbb{F}}$. Moreover, $\mathrm{Aut}^{+}(\mathbb{F})$ denotes the order-preserving automorphisms and $\mathrm{PLL}_{2}^{ \pm}$denotes the order-preserving and order-reversing transformations respectively, that is, $\mathrm{P}^{ \pm} L_{2}^{ \pm}=\Pi^{ \pm}(\overline{\mathbb{F}}) \cap$ $\mathrm{PCL}_{2}=\mathrm{PGL}_{2}^{ \pm} \operatorname{Aut}^{+}(\mathbb{F})$.

We furthermore introduce for later use the collection $\mathrm{Add}^{+}(\mathbb{F})$ of all additive orderpreserving permutations of $\mathbb{F}$ and the collection $\mathrm{Mul}^{+}(\mathbb{F})$ of all multiplicative orderpreserving permutations of $\mathbb{F}$ (both kinds of permutations extended canonically onto $\overline{\mathbb{F}})$. With these permutations we form the following subgroups of $\Pi^{+}(\overline{\mathbb{F}})$ :

$$
\begin{aligned}
& \Delta=\Delta(\mathbb{F})=\mathrm{PGL}_{2}^{+} \mathrm{Add}^{+}(\mathbb{F}) \mathrm{PGL}_{2}^{+} \\
& \Phi=\Phi(\mathbb{F})=\mathrm{PGL}_{2}^{+} \mathrm{Mul}^{+}(\mathbb{F}) \mathrm{PGL}_{2}^{+}
\end{aligned}
$$

Clearly, $\mathrm{PCL}_{2}^{+} \leq \Delta \cap \Phi$. In fact, equality holds as we shall see in Lemma 5.4.

We shall be using in particular the following permtations in $\mathrm{PGL}_{2}$ :

- the muliplication $\mu_{a}$ by $a \in \mathbb{F}, a \neq 0$, i.e., $\mu_{a}(x)=a x$. Obviously, $\mu_{a}$ is additive and $\mu_{a}$ is order-preserving if and only if $a>0$.

- the translation $\tau_{t}$ by $t \in \mathbb{F}$, i.e., $\tau_{t}(x)=x+t$. Clearly, $\tau_{t}$ always is orderpreserving. We furthermore denote by $\mathrm{T}(\mathbb{F})$ the collection of all translations by elements of $\mathbb{F}$, i.e.,

$$
\mathrm{T}=\mathrm{T}(\mathbb{F})=\{x \mapsto x+t \mid t \in \mathbb{F}\} \leq \mathrm{PGL}_{2} .
$$


Remark 2.5. Substituting $f$ and $g$ by $\delta f$ and $\delta^{\prime} g$, respectively, for two permutations $\delta, \delta^{\prime} \in \mathrm{PGL}_{2}^{+} \alpha$ where $\alpha \in \mathrm{Aut}^{+}(\mathbb{F})$, does not alter the circle set of the plane, that is, $\mathcal{M}(\mathbb{F} ; f, g)=\mathcal{M}\left(\mathbb{F} ; \delta f, \delta^{\prime} g\right)$. Since $\mathrm{PGL}_{2}^{+}$is still 2 -transitive and because the stabilizer $\left(\mathrm{PGL}_{2}^{+}\right)_{\infty, 0}$ of $\infty$ and 0 is transitive on $\mathbb{P}$, we may assume, if necessary, that $f$ and $g$ both fix $\infty, 0$ and 1 . We denote the stabilizers of $\infty, 0$ and 1 in $\Pi(\overline{\mathbb{F}})$ and $\Pi^{ \pm}(\overline{\mathbb{F}})$ by $\Pi_{\infty, 0,1}(\overline{\mathbb{F}})$ and $\Pi_{\infty, 0,1}^{ \pm}(\overline{\mathbb{F}})$ respectively.

2.6. Let $\mathcal{M}(\mathbb{F} ; f, g)$ be non-Miquelian and assume that $\mathbb{F} \not G F(9)$. (The Minkowski planes of order 9 play a special role, compare [19] for the corresponding situation in projective planes. For more details about Minkowski planes of order 9 see [3] and [16].) Then the automorphisms of $\mathcal{M}(\mathbb{F} ; f, g)$ that preserve (+)- and (-)-parallel classes are of the following form:

(1) $(x, y) \mapsto(\alpha(x), \beta(y))$ is an automorphism of $\mathcal{M}(\mathbb{F} ; f, g)$ that preserves $(+)$ and $(-)$-parallel classes and fixes each of the two components of the circle set if and only if

$$
\begin{aligned}
\alpha & \in \mathrm{PGL}_{2} \phi \cap f^{-1} \mathrm{PGL}_{2} \psi f \\
\beta & \in \mathrm{PGL}_{2} \phi \cap g^{-1} \mathrm{PGL}_{2} \psi g \\
\beta \alpha^{-1} & \in \mathrm{PGL}_{2}^{+}
\end{aligned}
$$

for $\phi, \psi \in \operatorname{Aut}^{+}(\mathbb{F})$.

(2) $(x, y) \mapsto(\alpha(x), \beta(y))$ is an automorphism of $\mathcal{M}(\mathbb{F} ; f, g)$ that preserves (+)and $(-)$-parallel classes and exchanges the two components of the circle set if and only if

$$
\begin{aligned}
\alpha & \in f^{-1} \mathrm{PGL}_{2} \phi \cap \mathrm{PGL}_{2} \psi f \\
\beta & \in g^{-1} \mathrm{PGL}_{2} \phi \cap \mathrm{PGL}_{2} \psi g \\
\beta \alpha^{-1} & \in g^{-1} \mathrm{PGL}_{2}^{-} f
\end{aligned}
$$

for $\phi, \psi \in \operatorname{Aut}^{+}(\mathbb{F})$.

For the next Lemma we follow [20, Lemma 4.2] which uses the group $\mathrm{L}_{2}^{+}(\mathbb{F})=\{x \mapsto$ $a x+t \mid a, t \in \mathbb{F}, a>0\} \leq \mathrm{PGL}_{2}^{+}$. (But we then obtain that $\alpha$ below is in $\operatorname{Aut}^{+}\left(\mathbb{F}^{-}\right)$.)

Lemma 2.7. Assume that $\phi \mathrm{T} \psi^{-1} \subseteq \mathrm{PGL}_{2}^{+}$for two permutations $\phi, \psi$ of $\overline{\mathbb{F}}$. Then $\phi, \psi \in \mathrm{PGL}_{2} \alpha$ for some additive permutation $\alpha \in \Pi^{+}(\mathbb{F})$. (The same $\alpha$ for both permutations.)

Proof. Replacing $\phi$ and $\psi$ by $\sigma \phi$ and $\tau \psi$, respectively, for two suitable permutations $\sigma, \tau \in \mathrm{PGL}_{2}$, we may assume that $\phi$ and $\psi$ both fix $\infty, 1$ and 0 . For each $t \in \mathbb{F}$ there then exist $a_{t}, b_{t}, c_{t}, d_{t} \in \mathbb{F}, a_{t} d_{t}-b_{t} c_{t} \neq 0$, such that

$$
\phi(x+t)=\frac{a_{t} \psi(x)+b_{t}}{c_{t} \psi(x)+d_{t}}
$$


for all $x \in \overline{\mathbb{F}}$. Evaluating both sides at $x=\infty$ and $x=0$ gives us $c_{t}=0$ and $b_{t} / d_{t}=\phi(t)$. For $t=0$ we then obtain $\phi(x)=\left(a_{0} / d_{0}\right) \psi(x)$. Evaluating at $x=1$ yields $a_{0} / d_{0}=1$ and thus $\phi=\psi$. Let $\alpha_{t}=a_{t} / d_{t}$. Then

$$
\phi(x+t)=\alpha_{t} \phi(x)+\phi(t)
$$

for all $x, t \in \mathbb{F}$. Since the left-hand side is symmetrical in $x$ and $t$, we find that

$$
\alpha_{t}=\gamma \phi(t)+1
$$

for some constant $\gamma \in \mathbb{F}$. Since $\alpha_{t} \neq 0$ for all $t \in \mathbb{F}$, we must have $\gamma=0$. (Otherwise $t_{0}=\phi^{-1}(-1 / \gamma)$ is defined and $\alpha_{t_{0}}=0$.) Hence $\alpha_{t}=1$ is constant. Therefore the restriction of $\phi$ to $\mathbb{F}$ is additive.

\section{Klein-Kroll Types of Minkowski Planes $\mathcal{M}(\mathbb{F} ; f, g)$ With RESPECT TO $G$-TRANSLATIONS}

Similar to the Lenz-Barlotti classification for projective planes Minkowski planes were classified with respect to $G$ - and $\{q\}$-translations in [8]; cf. [14] for a corresponding classification of finite Minkowski planes. Let $G$ be a parallel class (or generator) of a Minkowski plane $\mathcal{M}$. A $G$-translation of $\mathcal{M}$ is an automorphism of $\mathcal{M}$ that fixes precisely the points of $G$ or the identity. A group of $G$-translations of $\mathcal{M}$ is called $G$-transitive, if it acts transitively on each parallel class $H$ of type opposite the type of $G$ without the point of intersection with $G$. We say that the automorphism group $\Gamma$ of $\mathcal{M}$ is $G$-transitive if $\Gamma$ contains a $G$-transitive subgroup of $G$-translations.

With respect to $G$-translations M. Klein and H.-J. Kroll obtained six types of Minkowski planes, in fact, the more general hyperbola structures, see [8, Theorem 3.4]. If $\mathcal{Z}$ denotes the set of all parallel types $G$ for which the Minkowski plane is $G$ transitive, then exactly one of the following statements is valid:
A. $\mathcal{Z}=\emptyset$
B. $|\mathcal{Z}|=1$;
C. $\mathcal{Z}=\left\{|p|_{+},|p|_{-}\right\}$for some point $p$;
D. $\mathcal{Z}$ consists of all $(+)$-parallel classes or of all $(-)$-parallel classes;
E. $\mathcal{Z}$ consists of all $(+)$-parallel classes plus one $(-)$-parallel class or of all $(-)$ - parallel classes plus one $(+)$-parallel class;
F. $\mathcal{Z}$ consists of all $(+)$ - and all $(-)$-parallel classes.

Lemma 3.1. $\Gamma_{f, g}$ is $G$-transitive for some parallel class $G$ if and only if $f$ or $g$ belongs to $\Delta$, cf. 2.4 .

Proof. Suppose that $\mathcal{M}(\mathbb{F} ; f, g)$ is $G$-transitive for some generator $G$. Up to isomorphisms of type 2.1 and 2.2 we may assume that $G$ is the (-)-parallel class of the point $(\infty, \infty)$ and, by Remark 2.5 , that $f$ and $g$ both fix $\infty$. A $G$-translation then 
is of the form $(x, y) \mapsto(\alpha(x), \beta(y))$ where $\alpha=i d$, and $\beta$ is as in 2.6.(1) or 2.6.(2). The latter case cannot occur since by (FP) two circles that touch at a point are in the same component and because a $G$-translation takes a circle $K$ to one that touches $K$ at $K \cap G$.

In the former case we obtain

$$
\begin{array}{r}
i d=\alpha \in \mathrm{PGL}_{2}^{+} \phi \cap f^{-1} \mathrm{PGL}_{2}^{+} \psi f \quad \text { and } \\
\beta \in \mathrm{PGL}_{2}^{+} \phi \cap g^{-1} \mathrm{PGL}_{2}^{+} \psi g
\end{array}
$$

for $\phi, \psi \in$ Aut $^{+}$. Thus $\phi=\psi=i d$. Furthermore, $\beta \in \mathrm{PGL}_{2}^{+}$must fix precisely the point $\infty$. Hence $\beta$ must be of the form $\beta(y)=y+t$ for some $t \in \mathbb{F}$.

Each $\tau \in \mathrm{T}$, cf. 2.4 , is an automorphism of $\mathcal{M}(\mathbb{F} ; f, g)$ and so $g \mathrm{~T}^{-1} \subseteq \mathrm{P}_{2}^{+}$. By Lemma 2.7, $g$ must be the composition of a permutation in $\mathrm{PGL}_{2}$ and an additive permutation of $\mathbb{F}$. Hence $g \in \Delta$.

Conversely, suppose that $g \in \Delta$, that is, $g=\sigma \alpha \rho$ with $\sigma, \rho \in \mathrm{PGL}_{2}^{+}$and $\alpha \in$ $\operatorname{Add}^{+}(\mathbb{F})$. Then for each $t \in \mathbb{F}$ the mapping

$$
(x, y) \mapsto\left(x, \rho^{-1}(\rho(y)+t)\right)
$$

is an automorphism of $\mathcal{M}(\mathbb{F} ; f, g)$. (Note that $\alpha \tau_{t}=\tau_{\alpha(t)} \alpha$ where $\tau_{t}(y)=y+t$.) Furthermore, the collection of all theses automorphisms for $t \in \mathbb{F}$ is transitive on each (+)-parallel class without the point whose ordinate is $\rho^{-1}(\infty)$; each of the latter points is fixed by every such automorphism. Hence $\Gamma_{f, g}$ is $G$-transitive for $G=\overline{\mathbb{F}} \times\left\{\rho^{-1}(\infty)\right\}$.

Theorem 3.2. The automorphism group $\Gamma_{f, g}$ of a Minkowski plane $\mathcal{M}(\mathbb{F} ; f, g)$ is of class

$\mathrm{F}$ if and only if $f, g \in \mathrm{P \Gamma L}_{2}^{+}$;

$\mathrm{E}$ if and only if $f \in \mathrm{P}^{+} L_{2}^{+}, g \in \Delta \backslash \mathrm{P}_{2}^{+}$or $f \in \Delta \backslash \mathrm{P}_{2}^{+}, g \in \mathrm{P}_{2}^{+}$;

$\mathrm{D}$ if and only if $f \in \mathrm{P}_{2}^{+}, g \in \Pi^{+}(\overline{\mathbb{F}}) \backslash \Delta$ or $f \in \Pi^{+}(\overline{\mathbb{F}}) \backslash \Delta, g \in \mathrm{P}_{2}^{+}$;

$\mathrm{C}$ if and only if $f, g \in \Delta \backslash \mathrm{P}_{2}^{+}$;

$\mathrm{B}$ if and only if $f \in \Pi^{+}(\overline{\mathbb{F}}) \backslash \Delta, g \in \Delta \backslash \mathrm{P} L_{2}^{+}$or $f \in \Delta \backslash \mathrm{P}^{2} L_{2}^{+}, g \in \Pi^{+}(\overline{\mathbb{F}}) \backslash \Delta$;

A if and only if $f, g \in \Pi^{+}(\overline{\mathbb{F}}) \backslash \Delta$.

Proof. Suppose that $\Gamma_{f, g}$ is $G$-transitive for at least two (-)-parallel classes. According to [8, Theorem 3.4] $\Gamma_{f, g}$ must be $G$-transitive for every (-)-parallel class. As in the proof of Lemma 3.1 we see that $(x, y) \mapsto(x, \gamma(y))$ is an automorphism of $\mathcal{M}(\mathbb{F} ; f, g)$ for every $\gamma \in \mathrm{PGL}_{2}^{+}$that fixes precisely one or all points of $\overline{\mathbb{F}}$. Let $\Sigma$ be the subgroup of $\mathrm{PGL}_{2}^{+}$generated by all these permutations $\gamma$. In fact, $\Sigma \subseteq \operatorname{PSL}(2, \mathbb{F})$ (i.e., those linear fractional permutations $x \mapsto(a x+b) /(c x+d)$ with $a d-b c=1)$, and it is not hard to see that $\Sigma=\operatorname{PSL}(2, \mathbb{F})$. Then $g \Sigma g^{-1} \subseteq \mathrm{PGL}_{2}^{+}$by 2.6.(1). (Note that $\phi=\psi=i d$ in this case; cf. the proof of Lemma 3.1.)

We can assume that $g$ fixes $\infty, 1$ and 0 . Since $T=\{y \mapsto y+t \mid t \in \mathbb{F}\} \leq \operatorname{PSL}(2, \mathbb{F})$, we obtain that $g$ must be additive by Lemma 2.7. Furthermore, PSL $(2, \mathbb{F})$ contains 
all permutations of the form $x \mapsto a^{2} x$ and $x \mapsto-a^{2} / x$ for $a \neq 0$. From $g \gamma g^{-1} \in$ $\mathrm{PGL}_{2}^{+}$for all these permutations one finds the functional equations

$$
\begin{aligned}
g\left(a^{2} x\right) & =g\left(a^{2}\right) g(x) \quad \text { and } \\
g\left(-\frac{a^{2}}{x}\right) & =-\frac{g\left(a^{2}\right)}{g(x)}
\end{aligned}
$$

respectively. This shows that

$$
g(x y)=g(x) g(y)
$$

whenever at least one of $x, y$ or $x y$ is a square of $\mathbb{F}$. In particular, $g\left(x^{2}\right)=g(x)^{2}$ for all $x \in \mathbb{F}$. Therefore

$$
g(x y)^{2}=g\left((x y)^{2}\right)=g\left(x^{2} y^{2}\right)=g\left(x^{2}\right) g\left(y^{2}\right)=g(x)^{2} g(y)^{2}=(g(x) g(y))^{2} .
$$

Hence

$$
g(x y)= \pm g(x) g(y)
$$

for all $x, y \in \mathbb{F}$.

When $\mathbb{F}$ has characteristic 2 , then obviously $g$ is an automorphism of $\mathbb{F}$. If the characteristic of $\mathbb{F}$ is not 2 we define $H_{+}(x)=\{y \in \mathbb{F} \mid g(x y)=g(x) g(y)\}$ and $H_{-}(x)=\{y \in \mathbb{F} \mid g(x y)=-g(x) g(y)\}$. In fact, these two subsets of $\mathbb{F}$ are subgroups of the additive group of $\mathbb{F}$ and $\mathbb{F}=H_{+}(x) \cup H_{-}(x)$. This can only occur when $H_{+}(x)=\mathbb{F}$ or $H_{-}(x)=\mathbb{F}$. Since $1 \notin H_{-}(x)$ for $x \neq 0$ we must have $H_{+}(x)=\mathbb{F}$. This also holds true for $x=0$. Hence $g$ is multiplicative. Thus $g \in \operatorname{Aut}^{+}(\mathbb{F}) \subseteq$ $\mathrm{P}^{+} \mathrm{L}_{2}^{+}$.

Conversely, suppose that $g \in \mathrm{P \Gamma L}_{2}^{+}$. Then it readily follows that each mapping $(x, y) \mapsto(x, \gamma(y))$ is an automorphism of $\mathcal{M}(\mathbb{F} ; f, g)$ for each $\gamma \in \mathrm{PGL}_{2}^{+}$. (Note that each $\alpha \in$ Aut $^{+}(\mathbb{F})$ normalizes PGL $_{2}^{+}$.) In particular, each mapping $x \mapsto x+t$ for $t \in \mathbb{F}$ and all its conjugates in $\mathrm{PGL}_{2}^{+}$are automorphisms of $\mathcal{M}(\mathbb{F} ; f, g)$. This shows that $\Gamma_{f, g}$ is $G$-transitive for each (-)-parallel class.

Now the assertions of the theorem are an immediate consequence of Lemma 3.1 and the above considerations.

Various examples for types $A$ and $D$ can be found in $[6, \S 3]$, see also section 6 for examples of types F, D and A.

Corollary 3.3. $\Gamma_{f, g}$ is $G$-transitive for at least two parallel classes of the same type if and only if $f \in \mathrm{P \Gamma L}_{2}^{+}$or $g \in \mathrm{P \Gamma L}_{2}^{+}$.

Remark 3.4. A Minkowski plane $\mathcal{M}(\mathbb{F} ; f, g)$ of type $\mathrm{F}$ is isomorphic to $\mathcal{M}(\mathbb{F} ; \phi, i d)$ for some order-preserving automorphism $\phi$ of $\mathbb{F}$. Hence there are non-Miquelian Minkowski planes of type $\mathrm{F}$ in our family of planes if and only if $\mathbb{F}$ admits orderpreserving automorphisms $\neq i d$. 


\section{Klein-Kroll Types of Minkowski Planes $\mathcal{M}(\mathbb{F} ; f, g)$ With RESPECT TO $q$-TRANSLATIONS}

Let $q$ be a point of a Minkowski plane $\mathcal{M}$. A $q$-translation of $\mathcal{M}$ is an automorphism of $\mathcal{M}$ that is either the identity or fixes precisely the point $q$ and induces a translation of the derived affine plane $\mathcal{A}_{q}$ at $q$. More precisely, let $C$ be a circle passing through $q$. A $(q, C)$-translation of $\mathcal{M}$ is a $q$-translation that fixes $C$. A group of $(q, C)$ translations of $\mathcal{M}$ is called $(q, C)$-transitive, if it acts transitively on $C \backslash\{q\}$; a group of $q$-translation is called $q$-transitive, if it acts transitively on $P \backslash|q|$. We say that the automorphism group $\Gamma$ of $\mathcal{M}$ is $(q, C)$-transitive or $q$-transitive if $\Gamma$ contains a $(q, C)$-transitive subgroup of $(q, C)$-translations or a $q$-transitive subgroup of $q$ translations, respectively.

With respect to $q$-translations $M$. Klein and H.-J. Kroll obtained seven types of Minkowski planes see [8, Theorem 4.9]. If $Z$ denotes the set of all points $q$ for which the Minkowski plane is $(q, C)$-transitive for some circle $C$ through $q$, then exactly one of the following statements is valid:

I. $Z=\emptyset$;

II. $Z=\{q\}$ for some point $q$ and there is exactly one circle $C$ through $q$ such that $\mathcal{M}$ is $(q, C)$-transitive;

III. $Z=\{q\}$ for some point $q$ and $\mathcal{M}$ is $q$-transitive;

IV. $Z$ consists of the points on a circle;

V. $Z$ consists of the points on a parallel class;

VI. $Z=P$ and for each point $q$ there is exactly one circle $C$ through $q$ such that $\mathcal{M}$ is $(q, C)$-transitive;

VII. $Z=P$ and $\mathcal{M}$ is $q$-transitive for every point $q$.

In the following we need a rather technical condition. We only use it in the proof of Lemma 4.2; its other ocurences come from applying Lemma 4.2 . Let $G$ be a subgroup of the additive group of the half-ordered field $\mathbb{F}$. We say that an orderpreserving permutation $\phi$ of $\mathbb{F}$ is $G$-additive if and only if $\phi(x+y)=\phi(x)+\phi(y)$ for all $x \in \mathbb{F}$ and all $y \in G$. We then say that a half-ordered field $\mathbb{F}$ satifies (SA) if and only if

(SA) For every subgroup $G$ of the additive group of $\mathbb{F}$ such that the factor group $\mathbb{F} / G$ is isomorphic to a subgroup of $\mathrm{Aut}^{+}(\mathbb{F})$ and every $G$-additive monotonic permutation $\phi$ of $\mathbb{F}$ the subgroup $\phi(G)$ generates $\mathbb{F}$ as a field.

Roughly speaking, this condition means that $\phi(G)$ and thus $G$ cannot be too small and consequently $\mathrm{Aut}^{+}(\mathbb{F})$ cannot be too big as compared with the additive group of $\mathbb{F}$. ((SA.) stands for small automorphism group, or more precisely, a small abelian group of order-preserving automorphisms.) In fact, we can weaken (SA) a bit, but then it becomes even more technical. Note however that not every half-ordered field F satisfies (SA), see 4.1 .4 below for a counterexample.

Examples 4.1.

(1) For every half-ordered field ' $F$ for which $\operatorname{Aut}^{+}(\mathbb{F})$ is trivial we have $G=$ $\phi(G)=\mathbb{F}$ for each subgroup as in (SA). Hence each such field satisfies (SA). 
In particular, every subfield $\mathbb{F}$ of $\mathbb{R}$ with the familiar Euclidean ordering satisfies (SA) since $\mathrm{Aut}^{+}(\mathbb{F})$ is trivial in this case.

(2) Every finite field $\mathbb{F}$ satisfies (SA). Let $\mathbb{F}$ be of order $p^{n}$. The automorphism group of $\mathbb{F}$ is cyclic of order $n$ (generated by the Frobenius automorphism $x \mapsto x^{p}$ ). If the prime $p$ does not divide $n$, then Aut(F) contains no subgroup of order $p$. Hence $G=\mathbb{F}$ for each subgroup $G$ as in axiom (SA), and consequently $\phi(G)=\mathbb{F}$.

If $p$ divides $n$, then a subgroup $H=\phi(G)$ of index $p$ is possible besides $H=\mathbb{F}$ but no other indices can occur, since $\mathbb{F} / H$ is elementary abelian and $\operatorname{Aut}(\mathbb{F})$ is cyclic. In this case $|H|=p^{n-1}$. Obviously, the subfield $\mathbb{E}$ generated by $H$ can only have order $p^{n}$ or $p^{n-1}$. In the former case $\mathbb{E}=\mathbb{F}$. An easy computation shows that the latter case can only occur if $p=n=2$; but then $\mathbb{F}$ is not half-ordered. This proves that $H$ generates $\mathbb{F}$ as a field and (SA) is satisfied.

(3) Let $p$ be a prime and let $\mathbb{Z}_{p}$ be the prime field of order $p$. We let $\mathbb{F}=\mathbb{Z}_{p}(X)$ be the field of rational functions over $\mathbb{Z}_{p}$, that is, $\mathbb{F}$ is the quotient field of the polynomial ring $\mathbb{Z}_{p}[X]$; cf. $[13$, IV $]$. The elements of $\mathbb{F}$ are of the form $r(X) / s(X)$ where $r(X)$ and $s(X) \neq 0$ are polynomials; addition and multiplication are the familiar ones. For a nonzero rational function $t(X)=$ $r(X) / s(X) \in \mathbb{F}$ we define $t(X) \in \mathbb{P}$ if and only if $\operatorname{deg} r(X)-\operatorname{deg} s(X)$ is even where $\operatorname{deg} r(X)$ and $\operatorname{deg} s(X)$ are the familiar degrees of the polynomials $r(X), s(X) \neq 0$. Note that this definition is independent of the particular representation of an element of $\mathbb{F}(X)$. Then $\mathbb{P}$ is a subgroup of index 2 in the multiplicative group of $\mathbb{F}$ and $\mathbb{F}$ becomes a half-ordered field. Note that this definition also works for $p=2$.

The automorphism group of $\mathbb{F}$ is isomorphic to $\operatorname{PGL}\left(2, \mathbb{Z}_{p}\right)$, cf. [13, VIII, Exercise 1]. An element $\left(\begin{array}{ll}a & b \\ c & d\end{array}\right) \in \operatorname{PGL}\left(2, \mathbb{Z}_{p}\right)$ induces an automorphism of $\mathbb{F}$ by defining $t(X)$ being mapped to $t((a X+b) /(c X+d))$. We determine the order-preserving automorphisms. Since $X<0$ in $\mathbb{F}$ and because $X$ is mapped to $(a X+b) /(c X+d)$ we must have $a=0$ or $c=0((a X+$ $b) /(c X+d) \in \mathbb{P}$ if $a, c \neq 0)$. Likewise, if $a=0$ then $X+1$ is taken to $b /(c X+d)+1=(c X+b+d) /(c X+d)$ which is in $\mathbb{P}$. This shows that Aut ${ }^{+}(\mathbb{F})$ consists exactly of the automorphisms induced by substituting $X$ by $a X+b$, $a, b \in \mathbb{Z}_{p}, a \neq 0$. Hence an abelian subgroup of $\mathrm{Aut}^{+}(\mathbb{F})$ is isomorphic to either the additive group of $\mathbb{Z}_{p}$ or a subgroup of the multiplicative group of $\mathbb{Z}_{p}$. In particular, the order of such a group is at most $p$.

We now show that $\mathbb{F}$ satisfies (SA). Let $G$ be a subgroup of the additive group of $\mathbb{F}$ such that $\mathbb{F} / G$ is isomorphic to a subgroup of $\mathrm{Aut}^{+}(\mathbb{F})$. Hence, the index $(\mathbb{F}: G) \leq p$, and obviously only index 1 or $p$ can occur. If $G=\mathbb{F}$, there is nothing to prove. So we suppose that $G$ has index $p$ in $\mathbb{F}$. Let $\phi$ be a $G$-additive monotonic permutation of $\mathbb{F}$ and let $H=\phi(G)$. The $G$ additivity of $\phi$ implies that $H$ is a subgroup of index $p$ in $\mathbb{F}$. Furthermore, $H$ is a vector subspace over $\mathbb{Z}_{p}$ of $\mathbb{F}$ considered as a vector space over $\mathbb{Z}_{p}$ and $H$ has codimension 1 in $\mathbb{F}$. Clearly $H$ generates $\mathbb{F}$ as a field and (SA) 
is satisfied.

(4) The last example can be modified to yield half-ordered fields that do not satisfy (SA). I am indebted to $\mathrm{H}$. Kiechle for the following construction. Let $\mathbb{E}$ be an infinitely countable field of prime caharacteristic $p>0$ (e.g., the the field $\mathbb{Z}_{p}(X)$ from $\left.(3)\right)$ and let $\mathbb{F}=\mathbb{E}(X)$ be the field of rational functions over $\mathbb{E}$. As in (3) we say that a nonzero element $t(X)=r(X) / s(X) \in \mathbb{F}$ is positive if and only if $\operatorname{deg} r(X)-\operatorname{deg} s(X)$ is even. We now claim that the trivial subgroup $G=\{0\}$ and the identity $\phi=i d$ satisfy the hypothesis of condition (SA) but of course not the conclusion. Clearly, $\mathbb{F} / G$ is isomorphic to $\mathbb{F}$ which in turn is isomorphic, as an additive group, to $\mathbb{E}$ since both $\mathbb{E}$ and $\mathbb{F}$ have the same cardinality. Furthermore, $\mathbb{E}$ is canonically isomorphic to $\{t(X) \mapsto t(X+a) \mid a \in \mathbb{E}\}$ which is a subgroup of $\operatorname{Aut}^{+}(\mathbb{F})$.

Lemma 4.2. Let $\mathbb{F}$ be a half-ordered field that satisfies (SA). Then $\Gamma_{f, g}$ is $(q, C)$ transitive for some point $q$ and some circle $C$ through $q$ if and only if $f$ and $g$ belong to $\Delta$.

Proof. Suppose that $\mathcal{M}(\mathbb{F} ; f, g)$ is $(q, C)$-transitive for some point $q$ and some circle $C=K_{\gamma}$ through $q$. Up to an isomorphism of type 2.1 we may assume that $q=$ $(\infty, \infty)$ and using isomorphisms of types 2.1 and 2.3 , if necessary, we may further assume that $C=K_{i d}$; following Remark 2.5 we can achieve that $f$ and $g$ both fix $\infty, 0$ and 1 .

A $(q, C)$-translation then is of the form 2.6.(1), i.e., $(x, y)$ maps to $(\alpha(x), \beta(y))$ where

$$
\begin{aligned}
\alpha & \in \mathrm{PGL}_{2} \phi \cap f^{-1} \mathrm{PGL}_{2} \psi f \\
\beta & \in \mathrm{PGL}_{2} \phi \cap g^{-1} \mathrm{PGL}_{2} \psi g \\
\beta \alpha^{-1} & \in \mathrm{PGL}_{2}^{+}
\end{aligned}
$$

and $\phi, \psi \in \operatorname{Aut}^{+}(\mathbb{F})$.

The tangent bundle of $C$ at $q$ consists of all circle $K_{\tau_{t}}, t \in \mathbb{F}$, where $\tau_{t}$ is the translation by $t$ as in 2.4. Since each circle in this bundle is fixed, we obtain

$$
\beta \tau_{t}=\tau_{t} \alpha
$$

for all $t \in \mathbb{F}$. In particular, $t=0$ yields $\beta=\alpha$. Then

$$
\alpha(x+t)=\alpha(x)+t
$$

for all $t, x \in \mathbb{F}$. Evaluating at $x=0$ we find $\alpha(t)=\alpha(0)+t$, i.e.,

$$
\alpha=\tau_{\alpha(0)}
$$

Clearly, $\alpha \in \mathrm{PGL}_{2} \phi$ with $\phi=i d \in \operatorname{Aut}^{+}(\mathbb{F})$ and $i d=\beta \alpha^{-1} \in \mathrm{PGL}_{2}^{+}$.

The $(q, C)$-transitivity further yields that for each $a \in \mathbb{F}$ there is a corresponding $(q, C)$-translation and an $\alpha_{a}$ such that $\alpha_{a}(0)=a$. From above then $\alpha_{a}=\tau_{a}$. In order to obtain an automorphism the condition $\alpha_{a} \in f^{-1} \mathrm{PGL}_{2} \psi_{a} f$ for some $\psi_{a} \in$ 
Aut $^{+}(\mathbb{F})$ must still be satisfied. Hence, there is $\sigma_{a} \in \mathrm{PGL}_{2}$ such that $f \tau_{a}=\sigma_{a} \psi_{a} f$. This only implies that $f \mathrm{~T} f^{-1} \subseteq \mathrm{P}_{2}^{+}$so we cannot apply Lemma 2.7. Evaluating both sides at $\infty, 0$ and 1 one finds $\sigma_{a}(x)=(f(a+1)-f(a)) x+f(a)$. Therefore

$$
f(x+a)=(f(a+1)-f(a)) \psi_{a}(f(x))+f(a)
$$

for all $x, a \in \mathbb{F}$, or

$$
x \mapsto \frac{f\left(a+f^{-1}(x)\right)-f(a)}{f(a+1)-f(a)}
$$

is an automorphism of $\mathbb{F}$ for each $a \in \mathbb{F}$. (This is $\psi_{a}$ from above.)

Since the $(q, C)$-translations form a group, it readily follows that the map $\Psi: \mathbb{F} \rightarrow$ $\mathrm{Aut}^{+}(\mathbb{F})$ from the additive group of $\mathbb{F}$ to the group of order-preserving automorphisms of $\mathbb{F}$ defined by $\Psi(a)=\psi_{a}$ is a homomorphism of groups. Let $K$ denote the kernel of $\Psi$. For $a \in K$ we then have

$$
f(x+a)=(f(a+1)-f(a)) f(x)+f(a)
$$

for all $x \in \mathbb{F}$. Let $c=f(a+1)-f(a)$. Then

$$
f(x+a)=(c-1) f(x)+f(x)+f(a)
$$

for all $x \in \mathbb{F}$. If $c \neq 1$, there is an $x_{a} \in \mathbb{F}$ such that $(c-1) f\left(x_{a}\right)+f(a)=0$. But then $f\left(x_{a}+a\right)=f\left(x_{a}\right)$, and therefore $a=0$ because $f$ is a permutation of $\mathbb{F}$. Hence, if $a \neq 0$ we must have $c=1$ and

$$
f(x+a)=f(x)+f(a)
$$

for all $x \in \mathbb{F}$. This also holds for $a=0$. Hence $f$ is $K$-additive. Furthermore, $\mathbb{F} / K$ is isomorphic to a subgroup of $\mathrm{Aut}^{+}(\mathbb{F})$.

Let $x \in K$ and $a \in \mathbb{F}$. Then, using (2), identity (1) becomes

$$
f(x)+f(a)=f(x+a)=(f(a+1)-f(a)) \psi_{a}(f(x))+f(a) .
$$

Hence

$$
\psi_{a}(f(x))=\frac{f(x)}{f(a+1)-f(a)}
$$

or

$$
\psi_{a}(y)=c_{a} y
$$

for each $y \in f(K)$, where $c_{a}=(f(a+1)-f(a))^{-1}$.

Since $f(K)$ generates $\mathbb{F}$ by axiom (SA), there is at least one nonzero element $y_{0} \in$ $f(K)$. Let $f_{0}: \mathbb{F} \rightarrow \mathbb{F}$ be defined by $f_{0}(x)=f(x) y_{0}^{-1}$. Clearly, $f_{0}$ is a monotonic permutation of $\mathbb{F}$ and $f_{0}$ also is $K$-additive. Hence, $f_{0}(K)$ generates $\mathbb{F}$ by axiom (SA). However, for $y \in f_{0}(K), y=z y_{0}^{-1}$ for some $z \in f(K)$, we have

$$
\begin{aligned}
\psi_{a}(y) & =\psi_{a}\left(z y_{0}^{-1}\right)=\psi_{a}(z) \psi_{a}\left(y_{0}^{-1}\right) \\
& =c_{a} z\left(c_{a} y_{0}\right)^{-1}=z y_{0}^{-1} \\
& =y
\end{aligned}
$$


Thus $\left.\psi_{a}\right|_{f_{0}(K)}=i d$. Since $f_{0}(K)$ generates $\mathbb{F}$ as a field, we obtain that $\psi_{a}=i d$. This shows that $K=\mathbb{F}$, and consequently, $f$ is additive by (2).

Likewise, the condition $\alpha \in g^{-1} \mathrm{PGL}_{2} \psi g$ yields that $g$ is additive.

Note that $\gamma \Delta^{-1} \gamma^{-1}=\Delta$ so that under an isomorphism of type 2.3 a Minkowski plane $\mathcal{M}(\mathbb{F} ; f, g)$ with $f, g \in \Delta$ is taken to a Minkowski plane $\mathcal{M}\left(\mathbb{F} ; f^{\prime}, g^{\prime}\right)$ with $f^{\prime}, g^{\prime} \in \Delta$.

Conversely, suppose that $f, g \in \Delta$, that is, $f=\sigma_{1} \alpha_{1} \rho_{1}$ and $g=\sigma_{2} \alpha_{2} \rho_{2}$ with $\sigma_{i}, \rho_{i} \in \mathrm{PGL}_{2}^{+}$and $\alpha_{i} \in \mathrm{Add}^{+}$for $i=1,2$. Then for each $t \in \mathbb{F}$ the mapping

$$
(x, y) \mapsto\left(\rho_{1}^{-1}\left(\rho_{1}(x)+t\right), \rho_{2}^{-1}\left(\rho_{2}(y)+t\right)\right)
$$

is an automorphism of $\mathcal{M}(\mathbb{F} ; f, g)$. (Note that $\alpha_{i} \tau_{t}=\tau_{\alpha_{i}(t)} \alpha_{i}$.) Furthermore, each such automorphism for $t \in \mathbb{F}$ is a $\left(\left(\rho_{1}^{-1}(\infty), \rho_{2}^{-1}(\infty)\right), K_{\rho_{2}^{-1} \rho_{1}}\right)$-translation and the collection of all theses automorphisms for $t \in \mathbb{F}$ acts transitively on $K_{\rho_{2}^{-1} \rho_{1}}$ । $\left\{\left(\rho_{1}^{-1}(\infty), \rho_{2}^{-1}(\infty)\right)\right\}$. Hence $\Gamma_{f, g}$ is $\left(\left(\rho_{1}^{-1}(\infty), \rho_{2}^{-1}(\infty)\right), K_{\rho_{2}^{-1} \rho_{1}}\right)$-transitive.

Remark 4.3. By [1] every order-preserving permutation of a finite half-ordered field $\mathbb{F}$ of odd order is an affine transformation of an automorphism of $\mathbb{F}$. Hence in this case every order-preserving permutation of $\mathbb{F}$ that fixes 0 and 1 is an automorphism of $\mathbb{F}$ and thus additive.

Remark 4.4. The last part in the proof of Lemma 4.2 does not use property (SA). Hence $\Gamma_{f, g}$ is $(q, C)$-transitive for some point $q$ and some circle $C$ through $q$ if $f$ and $g$ belong to $\Delta$. Furthermore, if a permutation of $\overline{\mathbb{F}}$ belongs to $\mathrm{PLL}_{2}^{+}$, there are many ways to represent it in the form $\sigma \alpha \rho$ with $\sigma, \rho \in \mathrm{PGL}_{2}^{+}$and $\alpha \in \mathrm{Aut}^{+}(\mathbb{F})$. This implies that $\Gamma_{f, g}$ is $(q, C)$-transitive for every point $q$ and every circle $C$ through $q$ if $f, g \in \mathrm{PLL}_{2}^{+}$and that $\Gamma_{f, g}$ is $(q, C)$-transitive for every point $q$ on a parallel class and some circle $C$ through $q$ if $f \in \mathrm{PLL}_{2}^{+}$and $g \in \Delta$. In particular, $\Gamma_{f, g}$ is of class VII or at least $V$ in the respective cases.

Corollary 4.5. Let $\mathbb{F}$ be a half-ordered field that satisfies (SA) and let $q$ be a point of $\mathcal{M}(\mathbb{F} ; f, g)$. Then $\Gamma_{f, g}$ is $(q, C)$-transitive for some circle $C$ through $q$ if and only $\Gamma_{f, g}$ is q-transitive. In this case $\Gamma_{f, g}$ is $|q|_{--}$and $|q|_{+}$-transitive.

Proof. If $\Gamma_{f, g}$ is $q$-transitive, then it is $(q, C)$-transitive for every circle $C$ through $q$. Conversely, if $\Gamma_{f, g}$ is $(q, C)$-transitive for some circle $C$ through $q$, then both $f$ and $g$ belong to $\Delta$ by Lemma 4.2 . Therefore $\Gamma_{f, g}$ is $|q|_{--}$and $|q|_{+}$-transitive by Lemma 3.1. This implies the $q$-transitivity of $\Gamma_{f, g}$.

Theorem 4.6. Let $\mathbb{F}$ be a half-ordered field that satisfies (SA). Then the automorphism group $\Gamma_{f, g}$ of a Minkowski plane $\mathcal{M}(\mathbb{F} ; f, g)$ is of type

VII if and only if $f, g \in \mathrm{P \Gamma L}_{2}^{+}$;

$\mathrm{V}$ if and only if $f \in \mathrm{P}_{2}^{+}, g \in \Delta \backslash \mathrm{P}_{2}^{+}$or $f \in \Delta \backslash \mathrm{PLL}_{2}^{+}, g \in \mathrm{P \Gamma L}_{2}^{+}$;

III if and only if $f, g \in \Delta \backslash \mathrm{PCL}_{2}^{+}$

I if and only if $f \in \Pi^{+}(\overline{\mathbb{F}}) \backslash \Delta$ or $g \in \Pi^{+}(\overline{\mathbb{F}}) \backslash \Delta$. 
The types II, IV and VI do not occur among the Minkowski planes $\mathcal{M}(\mathbb{F} ; f, g)$.

Proof. By Corollary 4.5 the $(q, C)$-transitivity of $\Gamma_{f, g}$ implies its $q$-transitivity. Hence types II and VI cannot occur.

If $\Gamma_{f, g}$ is of type VII, then $\Gamma_{f, g}$ is $q$-transitive for each point $q$. Hence $\Gamma_{f, g}$ is $G$ transitive for each parallel class and so $\Gamma_{f, g}$ is of type F. By Theorem 3.2 therefore $f, g \in \mathrm{P \Gamma L}_{2}^{+}$.

If $\Gamma_{f, g}$ is of type $\mathrm{V}$, then $\Gamma_{f, g}$ is $q$-transitive for each point $q$ on a certain parallel

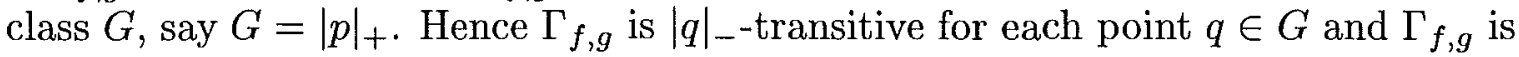
also $G$-transitive. Thus $\Gamma_{f, g}$ is of type $\mathrm{E}$ or $\mathrm{F}$. Therefore, by Theorem 3.2, at least one of $f$ or $g$ belongs to $\mathrm{P \Gamma L}_{2}^{+}$and the other to $\Delta$. If both belong to $\mathrm{PCL}_{2}^{+}$, then we have type VII. Hence we must have $f \in \mathrm{P}_{2}^{+}, g \in \Delta \backslash \mathrm{P}_{2}^{+}$or $f \in \Delta \backslash \mathrm{PLL}_{2}^{+}$, $g \in \mathrm{PTL}_{2}^{+}$.

Suppose that $\Gamma_{f, g}$ is of type IV. Then $\Gamma_{f, g}$ is $q$-transitive for each point on a certain circle. Hence $\Gamma_{f, g}$ is $G$-transitive for each parallel class and so $\Gamma_{f, g}$ is of type F. By Theorem 3.2 therefore $f, g \in \mathrm{P}_{2}^{+}$and $\Gamma_{f, g}$ is of type VII.

For examples of Minkowski planes of types VII and I see section 6.

Combining both classifications with respect to $G$-translations and with respect to $q$-translations M. Klein and H.-J. Krolll obtained the following ten types of Minkowski planes, see [8, Theorem 4.12]: I.A, I.B, I.D, II.A, III.C, IV.A, V.A, V.E, VI.A, VII.F. In view of Theorems 3.2 . and 4.6 one readily obtains that only six of these types can actually occur among our Minkowski planes and that those types can be characterised as follows.

Theorem 4.7. Let $\mathbb{F}$ be a half-ordered field that satisfies (SA). Then the automorphism group $\Gamma_{f, g}$ of a Minkowski plane $\mathcal{M}(\mathbb{F} ; f, g)$ is of type

VII.F if and only if $f, g \in \mathrm{P \Gamma L}_{2}^{+}$;

V.E if and only if $f \in \mathrm{P}_{2}^{+}, g \in \Delta \backslash \mathrm{P \Gamma L}_{2}^{+}$or $f \in \Delta \backslash \mathrm{P}_{2}^{+}, g \in \mathrm{P}_{2}^{+}$;

III.C if and only if $f, g \in \Delta \backslash \mathrm{PCL}_{2}^{+}$;

I.D if and only if $f \in \mathrm{P}^{2} \mathrm{~L}_{2}^{+}, g \in \Pi^{+}(\overline{\mathbb{F}}) \backslash \Delta$ or $f \in \Pi^{+}(\overline{\mathbb{F}}) \backslash \Delta, g \in \mathrm{P}^{2} L_{2}^{+}$;

I.B if and only if $f \in \Pi^{+}(\overline{\mathbb{F}}) \backslash \Delta, g \in \Delta \backslash \mathrm{P \Gamma L}_{2}^{+}$or $f \in \Delta \backslash \mathrm{P \Gamma L}_{2}^{+}, g \in \Pi^{+}(\overline{\mathbb{F}}) \backslash \Delta$;

I.A if and only if $f, g \in \Pi^{+}(\overline{\mathbb{F}}) \backslash \Delta$.

The types II.A, IV.A, V.A and VI.A do not occur among the Minkowski planes $\mathcal{M}(\mathbb{F} ; f, g)$.

\section{Klein-Kroll Types of Minkowski Planes $\mathcal{M}(\mathbb{F} ; f, g)$ With RESPECT TO $(p, q)$-HOMOTHETIES}

In this section we investigate a third kind of automorphisms which have been used in the classification in [7]. Let $p$ and $q$ be two nonparallel points of a Minkowski plane $\mathcal{M}$. An automorphism $\gamma$ of $\mathcal{M}$ is a $(p, q)$-homothety if $\gamma$ fixes $p$ and $q$ and induces a homothety with centre $q$ in the derived affine plane $\mathcal{A}_{p}$ at $p$. A group of $(p, q)$ homotheties is called $(p, q)$-transitive if it acts transitively on each circle through $p$ 
and $q$ minus the two points $p$ and $q$. We say that the automorphism group $\Gamma$ of $\mathcal{M}$ is $(p, q)$-transitive if $\Gamma$ contains a $(p, q)$-transitive subgroup of $(p, q)$-homotheties. With respect to $(p, q)$-homotheties $\mathrm{M}$. Klein obtained 23 types of Minkowski planes see [7, Theorem 2.15]. As remarked in [7, p. 127] the types 8, 9, 16 or 21 do not occur as types of the full automorphism group of a Minkowski plane, see also [12] for an amendment to type 9. (These types lead to Minkowski planes of order 3 or 4 and so the full automorphism group is of type 23.) Furthermore, H.-J. Kroll showed in [10] that type 22 cannot occur either. Finally, H.-J. Kroll and A. Madraś proved in [12, Corollary 2.7] and [11, Corollary 1] that there are no Minkowski planes of type 6 or 5 , respectively. Moreover they showed in [11] that under certain weak conditions Minkowski planes admitting automorphism groups of type 4 or 7 must be finite of order 3 or 5 (and then the Minkowski plane is Miquelian and of type 23). In fact, they show that the Minkowski planes $\mathcal{M}(\mathbb{F} ; f, g)$ satisfy their conditions and thus cannot be of type 4 or 7 , cf. [11, Corollary 2]. Since it easily follows that some of these types cannot occur for our Minkowski planes $\mathcal{M}(\mathbb{F} ; f, g)$, we included those types in Lemmata 5.1 and 5.2 .

Let $\mathcal{H}$ denote the set of all unordered pairs of points $\{p, q\}$ for which the Minkowski plane is $(p, q)$-transitive. Following we list only those types we shall refer to in this section in more detail:

1. $\mathcal{H}=\emptyset$

3. $\mathcal{H}=\left\{\{p, q\},\left\{|p|_{+} \cap|q|_{-},|p|_{-} \cap|q|_{+}\right\}\right\}$for some nonparallel points $p$ and $q$;

23. $\mathcal{H}$ consists of all pairs of nonparallel points.

The full automorphism groups of Miquelian Minkowski planes are of type 23, see $[7$, p. 126].

Given a permutation $h$ we can form the double coset $\mathrm{PGL}_{2}^{+} h \mathrm{PGL}_{2}^{+}$of the subgroup $\mathrm{PGL}_{2}^{+}$containing $h$. As with the usual cosets two double cosets of $\mathrm{PGL}_{2}^{+}$are either disjoint or identical. Note that every double coset $\mathrm{PGL}_{2}^{+} f \mathrm{PGL}_{2}^{+}$equals the single coset $f \mathrm{PGL}_{2}^{+}$for each $f \in \mathrm{P}^{2} \mathrm{~L}_{2}^{+}$.

Using this notation we can characterise $(p, q)$-transitivity as follows.

Lemma 5.1. $\Gamma_{f, g}$ is $(p, q)$-transitive for some nonparallel points $p, q$ if and only if $f, g \in \Phi$, cf. 2.4, and $f$ and $g$ belong to the same double coset of $\mathrm{PGL}_{2}^{+}$, that is, one has that $\mathrm{PGL}_{2}^{+} f \mathrm{PGL}_{2}^{+}=\mathrm{PGL}_{2}^{+} g \mathrm{PGL}_{2}^{+}$. (Equivalently, $f$ and $g$ both belong to $\mathrm{PGL}_{2}^{+} \mu \mathrm{PGL}_{2}^{+}$for some multiplicative order-preserving permutation $\mu$ of $\mathbb{F}$.)

Moreover, if $\Gamma_{f, g}$ is $(p, q)$-transitive, then $\Gamma_{f, g}$ is also $\left(|p|_{+} \cap|q|_{-},|p|_{-} \cap|q|_{+}\right)$transitive. In particular, $\Gamma_{f, g}$ is not of type $2,5,6,10,15,17,18$ or 20.

Proof. Suppose that $\mathcal{M}(\mathbb{F} ; f, g)$ is $(p, q)$-transitive for some points $p, q$. Up to an isomorphism of type 2.1 we may assume that $p=(\infty, \infty)$ and $q=(0,0)$; following Remark 2.5 we can achieve that $f$ and $g$ both fix $\infty, 0$ and 1 .

Every $((\infty, \infty),(0,0))$-homothety $\delta$ is of the form 2.6.(1) and every circle through $(\infty, \infty)$ and $(0,0)$ is fixed. The bundle of circles through $(\infty, \infty)$ and $(0,0)$ consists of the following circles

$$
\left\{K_{\mu_{r}} \mid r \in \mathbb{F}, r>0\right\} \cup\left\{K_{g^{-1} \mu_{s} f} \mid s \in \mathbb{F}, s<0\right\}
$$


where $\mu_{a}$ denotes muliplication by $a$, see 2.4 . If $\delta(x, y)=(\alpha(x), \beta(y))$, then

$$
\beta \mu_{r}=\mu_{r} \alpha
$$

and

$$
\beta g^{-1} \mu_{s} f=g^{-1} \mu_{s} f \alpha
$$

for all $r, s \in \mathbb{F}, r>0, s<0$. In particular, for $r=1$ one obtains $\alpha=\beta$. Then

$$
\alpha(r x)=r \alpha(x)
$$

for all $r, x \in \mathbb{F}, r>0$. Evaluating both sides at $x=1$ gives us $\alpha(r)=r \alpha(1)$ for all $r>0$; evaluating both sides at a fixed negative element $n$ gives us $\alpha(r n)=r \alpha(n)$ for all $r>0$. Hence

$$
\alpha(x)=\left\{\begin{array}{ll}
\alpha(1) x, & \text { if } x \geq 0 \\
(\alpha(n) / n) x, & \text { if } x<0
\end{array} .\right.
$$

But $\alpha \in \mathrm{PGL}_{2}^{+}$Aut $^{+}(\mathbb{F})$ by 2.6.(1), i.e., there are $\sigma \in \mathrm{PGL}_{2}^{+}$and $\phi \in \mathrm{Aut}^{+}(\mathbb{F})$ such that $\alpha=\sigma \phi$. Since $\alpha$ fixes 0 and $\infty$, we must have $\sigma=\mu_{a}$, where $a=\alpha(1)$. Then

$$
\phi(x)=\left\{\begin{array}{ll}
x, & \text { if } x \geq 0 \\
\frac{\alpha(n)}{n \alpha(1)} x, & \text { if } x<0
\end{array} .\right.
$$

Let $c=\alpha(n) /(n \alpha(1))$. Then

$$
n^{2}=\phi\left(n^{2}\right)=(\phi(n))^{2}=(c n)^{2}=c^{2} n^{2} .
$$

Hence $c^{2}=1$, and so $c= \pm 1$. If $c=1$, then $\phi=i d$ and $\alpha=\mu_{\alpha(1)}$. Suppose that $c=-1$. Then

$$
1-n=\phi(1)+\phi(n)=\phi(1+n)=\left\{\begin{array}{ll}
1+n, & \text { if } 1+n \geq 0 \\
-1-n, & \text { if } 1+n<0
\end{array}\right. \text {. }
$$

Therefore $2 n=0$ or $2=0$, respectively. In any case, $\mathbb{F}$ has characteristic 2 and $c=1$ in $\mathbb{F}$. Hence $\phi=i d$ and $\alpha=\mu_{m}$ where $m=\alpha(1)$.

From 2.6.(1) we further know that $\alpha \in f^{-1} \mathrm{PGL}_{2} \psi f \cap g^{-1} \mathrm{PGL}_{2} \psi g$ for some $\psi \in$ Aut $^{+}(\mathbb{F})$. Therefore, $f \mu_{m} f^{-1}=\rho \psi$ for some $\rho \in \mathrm{PGL}_{2}$. But $f \mu_{m} f^{-1}$ fixes 0 and $\infty$, so $\rho=\mu_{b}$ for some $b \in \mathbb{F}$. Evaluating at 1 yields $b=f(m)$. Hence

$$
f \mu_{m} f^{-1}=\mu_{f(m)} \psi
$$

and likewise,

$$
g \mu_{m} g^{-1}=\mu_{g(m)} \psi
$$

We still have not used the fact that every circle in the other half of the bundle through $(\infty, \infty)$ and $(0,0)$ is fixed by $\delta$. From above we find that $\mu_{m} g^{-1} \mu_{s} f=$ $g^{-1} \mu_{s} f \mu_{m}$ for all $s<0$. Thus

$$
\mu_{g(m)} \mu_{\psi(s)} \psi=\mu_{g(m)} \psi \mu_{s}=g \mu_{m} g^{-1} \mu_{s}=\mu_{s} f \mu_{m} f^{-1}=\mu_{s} \mu_{f(m)} \psi
$$


and therefore $g(m) \psi(s)=s f(m)$ for all $s<0$, i.e.,

$$
\psi(s)=\frac{f(m)}{g(m)} s
$$

for all $s<0$. Let $\lambda=f(m) / g(m)$. Then

$$
\lambda s r=\psi(s r)=\psi(s) \psi(r)=\lambda s \psi(r)
$$

for all $s<0, r \geq 0$. Hence

$$
\psi(x)=\left\{\begin{array}{ll}
x, & \text { if } x \geq 0 \\
\lambda x, & \text { if } x<0
\end{array} .\right.
$$

It follows as before that $\lambda=1$ and $\psi=i d$. Furthermore, $f=g$. Now $f \mu_{m} f^{-1}=$ $\mu_{f(m)}$ becomes

$$
f(m x)=f(m) f(x) .
$$

This identity holds for all $x \in \mathbb{F}$ and by the $((\infty, \infty),(0,0))$-transitivity also for all $m \in \mathbb{F}, m \neq 0$. Obviously, the above identity is also satisfied for $m=0$. This shows that $f=g$ is multiplicative.

Conversely, suppose that $f, g \in \mathrm{PGL}_{2}^{+} \mu \mathrm{PGL}_{2}^{+}$for some multiplicative order-preserving permutation $\mu$ of $\mathbb{F}$, that is, $f=\sigma_{1} \mu \rho_{1}$ and $g=\sigma_{2} \mu \rho_{2}$ with $\sigma_{i}, \rho_{i} \in \mathrm{PGL}_{2}^{+}$ for $i=1,2$. Then each mapping

$$
(x, y) \mapsto\left(\rho_{1}^{-1}\left(m \rho_{1}(x)\right), \rho_{2}^{-1}\left(m \rho_{2}(y)\right)\right)
$$

for $m \in \mathbb{F}, m \neq 0$, is an automorphism of $\mathcal{M}(\mathbb{F} ; f, g)$. (Note that $\mu \mu_{m}=\mu_{\mu(m)} \mu$ where $\mu_{m}(x)=m x$.) Furthermore, each such automorphism for $m \in \mathbb{F}, m \neq$ 0 , is a $(p, q)$-homothety where $p=\left(\rho_{1}^{-1}(\infty), \rho_{2}^{-1}(\infty)\right)$ and $q=\left(\rho_{1}^{-1}(0), \rho_{2}^{-1}(0)\right)$, and the collection of all theses automorphisms for $m \in \mathbb{F}, m \neq 0$, acts transitively on every circle through $p$ and $q$ minus these two points. Hence $\Gamma_{f, g}$ is $\left(\left(\rho_{1}^{-1}(\infty), \rho_{2}^{-1}(\infty)\right),\left(\rho_{1}^{-1}(0), \rho_{2}^{-1}(0)\right)\right.$-transitive.

Let $f=g=\mu$ be a multiplicative order-preserving permutation of $\mathbb{F}$ so that $\Gamma_{f, g}$ is $((\infty, \infty),(0,0))$-transitive. Let $\sigma \in \mathrm{PGL}_{2}^{+}$be defined by $\sigma(x)=-\frac{1}{x}$ for $x \in \mathbb{F}$, $x \neq 0$, and $\sigma(0)=\infty, \sigma(\infty)=0$. Then $\sigma \mu \sigma=\mu$, since $\mu$ is multiplicative. Hence, $\mathcal{M}(\mathbb{F} ; f, g)=\mathcal{M}(\mathbb{F} ; \sigma \mu \sigma, \mu)$ and $\Gamma_{f, g}$ is $\left(\left(\sigma^{-1}(\infty), \infty\right),\left(\sigma^{-1}(0), 0\right)\right.$-transitive by the above, that is, $\Gamma_{f, g}$ is $((0, \infty),(\infty, 0))$-transitive. The general case of $(p, q)$ transitivity readily follows from the above.

Finally, one readily checks that the sets $\mathcal{H}$ in types $2,5,6,10,15,17,18$ or 20 are not closed under the mapping $\{p, q\} \mapsto\left\{|p|_{+} \cap|q|_{-},|p|_{-} \cap|q|_{+}\right\}$. Hence these types cannot occur.

Lemma 5.2. Let $p, q, r$ be three points such that $q$ and $r$ are parallel but neither $q$ nor $r$ is parallel to $p$. Assume thät $\Gamma_{f, g}$ is $(p, q)$-transitive and $(p, r)$-transitive. Then $\Gamma_{f, g}$ is $G$-transitive for at least two parallel types of the same type and $\Gamma_{f, g}$ is 
of type 23. In particular, there is no Minkowski plane $\mathcal{M}(\mathbb{F} ; f, g)$ for which $\Gamma_{f, g}$ is of type 11, 12, 13, 14, 19 or 22 .

Proof. Let $p, q, r$ be three points as in the Lemma and let $q$ and $r$ be $(+)$-parallel. Then, by $[7,2.4], \Gamma_{f, g}$ is $|q|_{+}$-transitive. From Lemma 5.1 we further know that $\Gamma_{f, g}$ is also $\left(|p|_{+} \cap|q|_{-},|p|_{-} \cap|q|_{+}\right)$-transitive and $\left(|p|_{+} \cap|r|_{-},|p|_{-} \cap|r|_{+}\right)$-transitive. Since $|p|_{-} \cap|q|_{+}=|p|_{-} \cap|r|_{+}$and $|p|_{+} \cap|q|_{-}$is (+)-parallel to $|p|_{+} \cap|q|_{-}$, we obtain again by $[7,2.4]$ that $\Gamma_{f, g}$ is $|p|_{+}$-transitive. Hence $\Gamma_{f, g}$ is $G$-transitive for the two parallel classes $|p|_{+}$and $|q|_{+}$. From Corollary 3.3 it then follows that $f \in \mathrm{P} L_{2}^{+}$or $g \in \mathrm{PLL}_{2}^{+}$. (In fact, $g \in \mathrm{P}_{2}^{+}$by our choice of parallel classes.) Since $\Gamma_{f, g}$ is $(p, q)$-transitive, we have $\mathrm{PGL}_{2}^{+} f \mathrm{PGL}_{2}^{+}=\mathrm{PGL}_{2}^{+} g \mathrm{PGL}_{2}^{+}$by Lemma 5.1. However, this double coset equals $\mathrm{PCL}_{2}^{+}$. Therefore, $f, g \in \mathrm{P \Gamma L}_{2}^{+}$, and more precisely, $f, g \in \mathrm{PGL}_{2}^{+} \alpha$ for some order-preserving automorphism $\alpha$. This describes the Miquelian Minkowski plane, which is of type 23. The case that $q$ and $r$ is $(-)$-parallel is dealt with analogously.

Finally, one readily checks that the sets $\mathcal{H}$ in types $11,12,13,14,19$ or 22 all contain a triple of points $p, q, r$ as in the Lemma. Hence these types cannot occur.

After these preparations we can now classify our Minkowski planes with respect to $(p, q)$-homotheties.

Theorem 5.3. The automorphism group $\Gamma_{f, g}$ of a Minkowski plane $\mathcal{M}(\mathbb{F} ; f, g)$ is of type

23 if and only if $f, g \in \mathrm{P}_{2}^{+}$and $f \mathrm{PGL}_{2}^{+}=g \mathrm{PGL}_{2}^{+}$;

3 if and only if $f, g \in \Phi \backslash \mathrm{P}_{2}^{+}$and $f$ and $g$ are in the same double coset of $\mathrm{PGL}_{2}^{+}$;

1 if and only if $f \in \Pi^{+}(\overline{\mathbb{F}}) \backslash \Phi$ or $g \in \Pi^{+}(\overline{\mathbb{F}}) \backslash \Phi$ or $f, g \in \Phi$ but $f$ and $g$ are in different double cosets of $\mathrm{PGL}_{2}^{+}$, that is, $\mathrm{PGL}_{2}^{+} f \mathrm{PGL}_{2}^{+} \neq \mathrm{PGL}_{2}^{+} g \mathrm{PGL}_{2}^{+}$.

Proof. By the remarks made at the beginning of this section and by Lemmata 5.1 and 5.2 only types 1,3 and 23 can occur as types for the automorphism groups of our Minkowski planes.

The assertion of the theorem about type 1, i.e., there is no pair of points for which $\Gamma_{f, g}$ is $(p, q)$-transitive, readily follows from Lemma 5.1. Furthermore, if $\Gamma_{f, g}$ is of type 3 , then $f, g \in \Phi$ and $f$ and $g$ are in the same double coset of $\mathrm{PGL}_{2}^{+}$by Lemma 5.1. Suppose that $f \in \mathrm{P}_{2}^{+}$. Then $\mathrm{PGL}_{2}^{+} f \mathrm{PGL}_{2}^{+}=f \mathrm{PGL}_{2}^{+} \subseteq \mathrm{P}_{2}^{+}$and thus $g \in \mathrm{PGL}_{2}^{+} g \mathrm{PGL}_{2}^{+}=\mathrm{PGL}_{2}^{+} f \mathrm{PGL}_{2}^{+} \subseteq \mathrm{P \Gamma L}_{2}^{+}$. Hence $f, g \in \mathrm{P}_{2}^{+}$and, more precisely, $f, g \in \mathrm{PGL}_{2}^{+} \alpha$ for some order-preserving automorphism $\alpha$. Therefore, in this case, $\mathcal{M}(\mathbb{F} ; f, g)$ is Miquelian and $\Gamma_{f, g}$ is of type 23 . The case that $g \in \mathrm{P}^{2} L_{2}^{+}$is dealt with similarly. This shows that $\Gamma_{f, g}$ is of type 3 if and only if $f, g \in \Phi \backslash \mathrm{P}_{2}^{+}$ and $f$ and $g$ are in the same double coset of $\mathrm{PGL}_{2}^{+}$.

Types 1 and 3 do not cover only one case, namely, if $f, g \in \mathrm{PCL}_{2}^{+}$and $f \mathrm{PGL}_{2}^{+}=$ $g \mathrm{PGL}_{2}^{+}$. From what we said before it is clear that this case describes the Miquelian Minkowski plane, which is of type 23. 
Each of the above types actually occurs as the type of the full automorphism group of a Minkowski plane $\mathcal{M}(\mathbb{F} ; f, g)$ for suitable half-ordered fields $\mathbb{F}$ and suitable orderpreserving permutatison $f$ and $g$, see section 6 .

Combining both classifications from [8, Theroem 4.12] and [7, Theorem 2.15] M. Klein obtained 32 types of Minkowski planes see [7, Theorem 2.16]. In view of Theorem 4.7 and Theorem 5.3 and the restrictions given in [7, Theorem 2.16] of these 32 types only types VII.F.23, VII.F.1, V.E.1, III.C.1, I.D.1, I.B.1, I.A.3 and I.A.1 are possible for the automorphism groups of our Minkowski planes.

Before we can characterise the above mentioned types we investigate in the following lemma the possible intersection between $\Phi$ and $\Delta$.

Lemma 5.4. $\Phi \cap \Delta=\mathrm{P \Gamma L}_{2}^{+}$.

Proof. Obviously $\mathrm{P \Gamma L}_{2}^{+}$is contained in both $\Phi$ and $\Delta$. Let $f \in \Phi \cap \Delta$. Then there are $\alpha \in \operatorname{Add}^{+}(\mathbb{F}), \beta \in \mathrm{Mul}^{+}(\mathbb{F})$ and $\sigma_{1}, \sigma_{2}, \rho_{1}, \rho_{2} \in \mathrm{PGL}_{2}^{+}$such that $f=\sigma_{1} \alpha \rho_{1}=$ $\sigma_{2} \beta \rho_{2}$. Hence

$$
\sigma \alpha=\beta \rho
$$

where $\sigma=\sigma_{2}^{-1} \sigma_{1}, \rho=\rho_{2} \rho_{1}^{-1} \in \mathrm{PGL}_{2}^{+}$. We consider three cases depending on the form of $\sigma$ :

Case 1: $\sigma$ fixes $\infty$.

Then $\rho$ fixes $\infty$ too. We therefore have an identity

$$
a \alpha(x)+b=\beta(c x+d)
$$

for all $x \in \mathbb{F}$ and some $a, b, c, d \in \mathbb{F}, a, c>0$. There is a $b^{\prime} \in \mathbb{F}$ such that $b=a \alpha\left(b^{\prime}\right)$. By the additivity of $\alpha$ we find $a \alpha\left(x+b^{\prime}\right)=\beta(c x+d)$ for all $x \in \mathbb{F}$ and thus $a \alpha(x)=\beta\left(c x+d-c b^{\prime}\right)$ for all $x \in \mathbb{F}$. Evaluating at $x=0$ gives us $d-c b^{\prime}=0$ and thus

$$
a \alpha(x / c)=\beta(x)
$$

for all $x \in \mathbb{F}$. Since the left-hand side is additive in $x$, we obtain that $\beta \in \operatorname{Mul}^{+}(\mathbb{F}) \cap$ $\operatorname{Add}^{+}(\mathbb{F})=\operatorname{Aut}^{+}(\mathbb{F})$. This shows that $f \in \mathrm{PrL}_{2}^{+}$in this case.

Case 2: $\sigma$ maps $\infty$ to 0 .

Then $\rho$ also maps $\infty$ to 0 . We therefore have an identity

$$
\frac{a}{\alpha(x)+b}=\beta\left(\frac{c}{x+d}\right)
$$

for all $x \in \overline{\mathbb{F}}$ and some $a, b, c, d \in \mathbb{F}, a b, c d>0$. Taking reciprocals on both sides and using the multiplicity of $\beta$ we find $(\alpha(x)+b) / a=\beta(x+d) / \beta(c)$. This is an identity as in case 1 and it follows $f \in \mathrm{P \Gamma L}_{2}^{+}$.

Case 3: $\sigma$ maps $\infty$ to $r \neq 0, \infty$.

Then $\rho$ also maps $\infty$ to a point $\neq 0, \infty$. $\sigma$ has the form $\sigma(x)=(r x+a) /(x+b)$ and similarly $\rho(x)=(s x+c) /(x+d)$ for all $x \in \overline{\mathbb{F}}$ and some $r, s, a, b, c, d \in \mathbb{F}$, $r b-a, s d-c>0$. There is a $b^{\prime} \in \mathbb{F}$ such that $b=\alpha\left(b^{\prime}\right)$. By the additivity of $\alpha$ we find

$$
\frac{r \alpha(x)+a}{\alpha\left(x+b^{\prime}\right)}=\beta\left(\frac{s x+c}{x+d}\right)
$$


for all $x \in \overline{\mathbb{F}}$ and thus $\left(r \alpha(x)+a-r \alpha\left(b^{\prime}\right)\right) / \alpha(x)=\beta\left(\left(s x+c-s b^{\prime}\right) /\left(x+d-b^{\prime}\right)\right)$ for all $x \in \overline{\mathbb{F}}$. Let $a^{\prime}=a-r \alpha\left(b^{\prime}\right), c^{\prime}=c-s b^{\prime}$ and $d^{\prime}=d-b^{\prime}$. Then the above identity becomes

$$
\frac{r \alpha(x)+a^{\prime}}{\alpha(x)}=\beta\left(\frac{s x+c^{\prime}}{x+d^{\prime}}\right)
$$

for all $x \in \overline{\mathbb{F}}$. Evaluating at $x=0$ gives us $d^{\prime}=0$; evaluating at $x=-c^{\prime} / s$ gives us $r \alpha\left(-c^{\prime} / s\right)+a^{\prime}=0$ and thus $r \alpha\left(x+\left(c^{\prime} / s\right)\right) / \alpha(x)=\beta\left(\left(s x+c^{\prime}\right) / x\right)=\beta\left(s x+c^{\prime}\right) / \beta(x)=$ $\beta(s) \beta\left(x+\left(c^{\prime} / s\right)\right) / \beta(x)$ for all $x \in \overline{\mathbb{F}}$. Let $p=\beta(s) / r$ and $q=c^{\prime} / s$. By the additivity of $\alpha$ we obtain

$$
\alpha(x)=\alpha(q) \frac{\beta(x)}{p \beta(x+q)-\beta(x)}
$$

for all $x \in \overline{\mathbb{F}}$. Substitution of $x-q$ for $x$ and the additivity of $\alpha$ give us

$$
\alpha(q) \frac{\beta(x-q)}{p \beta(x)-\beta(x-q)}+\alpha(q)=\alpha(x-q)+\alpha(q)=\alpha(x)=\alpha(q) \frac{\beta(x)}{p \beta(x+q)-\beta(x)} .
$$

Hence

$$
\frac{p \beta(x)}{p \beta(x)-\beta(x-q)}=\frac{\beta(x)}{p \beta(x+q)-\beta(x)}
$$

and therefore

$$
p^{2} \beta(x+q)-2 p \beta(x)+\beta(x-q)=0
$$

for all $x \in \mathbb{F}$. Substitution of $q^{2} / x$ for $x$ in this identity and multiplication by $\beta(x) / \beta(q)$ yields

$$
p^{2} \beta(x+q)-2 p \beta(q)-\beta(x-q)=0
$$

Hence

$$
2 p^{2} \beta(x+q)-2 p(\beta(x)+\beta(q))=0
$$

and therefore

$$
p \beta(x+q)=\beta(x)+\beta(q)
$$

for all $x \in \mathbb{F}$. This finally gives us for $\alpha$

$$
\alpha(x)=\frac{\alpha(q)}{\beta(q)} \beta(x)
$$

or

$$
\beta(x)=\frac{\beta(q)}{\alpha(q)} \alpha(x)
$$

for all $x \in \mathbb{F}$. This shows that $\beta$ is additive. Thus $\beta \in \mathrm{P}^{+} \mathrm{L}_{2}^{+}$.

Combining Theorems 4.7 and 5.3 one readily obtains with Lemma 5.4 the following characterisation of the Klein-Kroll types of our Minkowski planes in terms of $f$ and $g$ as follows. 
Classification Theorem 5.5. Let $\mathbb{F}$ be a half-ordered field that satisfies (SA). Let

$$
\begin{aligned}
\Delta & =\mathrm{PGL}_{2}^{+} \mathrm{Add}^{+}(\mathbb{F}) \mathrm{PGL}_{2}^{+} \\
\Phi & =\mathrm{PGL}_{2}^{+} \mathrm{Mul}^{+}(\mathbb{F}) \mathrm{PGL}_{2}^{+}
\end{aligned}
$$

be as in 2.4 where $\mathrm{Add}^{+}(\mathbb{F})$ and $\mathrm{Mul}^{+}(\mathbb{F})$ denote the collections of all additive or multiplicative order-preserving permutations of $\mathbb{F}$, respectively. Then the automorphism group $\Gamma_{f, g}$ of a Minkowski plane $\mathcal{M}(\mathbb{F} ; f, g)$ is of type

VII.F.23 if and only if $f, g \in \mathrm{PCL}_{2}^{+}$and $f \mathrm{PGL}_{2}^{+}=g \mathrm{PGL}_{2}^{+}$(Miquelian Minkowski planes);

VII.F.1 if and only if $f, g \in \mathrm{P}^{+} \mathrm{L}_{2}^{+}$and $f \mathrm{PGL}_{2}^{+} \neq g \mathrm{PGL}_{2}^{+}$;

V.E.1 if and only if $f \in \mathrm{P} L L_{2}^{+}, g \in \Delta \backslash \mathrm{P}^{+} L_{2}^{+}$or $f \in \Delta \backslash \mathrm{P}_{2}^{+}, g \in \mathrm{P}_{2}^{+}$;

III.C. 1 if and only if $f, g \in \Delta \backslash \mathrm{P}_{2}^{+}$

I.D.1 if and only if $f \in \mathrm{P}^{2} \mathrm{~L}_{2}^{+}, g \in \Pi^{+}(\overline{\mathbb{F}}) \backslash \Delta$ or $f \in \Pi^{+}(\overline{\mathbb{F}}) \backslash \Delta, g \in \mathrm{P}^{+} L_{2}^{+}$;

I.B.1 if and only if $f \in \Pi^{+}(\overline{\mathbb{F}}) \backslash \Delta, g \in \Delta \backslash \mathrm{P}_{2}^{+}$or $f \in \Delta \backslash \mathrm{PLL}_{2}^{+}, g \in$ $\Pi^{+}(\overline{\mathbb{F}}) \backslash \Delta$;

I.A.3 if and only if $f, g \in \Phi \backslash \mathrm{P \Gamma L}_{2}^{+}$and $f$ and $g$ belong to the same double coset of $\mathrm{PGL}_{2}^{+}$;

I.A.1 if and only if $f, g \in \Pi^{+}(\overline{\mathbb{F}}) \backslash \Delta$ and one of the following holds:

- $f \in \Pi^{+}(\overline{\mathbb{F}}) \backslash \Phi$, or

$-g \in \Pi^{+}(\overline{\mathbb{F}}) \backslash \Phi$, or

- $f, g \in \Phi$ and $f$ and $g$ belong to different double cosets of $\mathrm{PGL}_{2}^{+}$, that is, $\mathrm{PGL}_{2}^{+} f \mathrm{PGL}_{2}^{+} \neq \mathrm{PGL}_{2}^{+} g \mathrm{PGL}_{2}^{+}$.

No other types can occur among the Minkowski planes $\mathcal{M}(\mathbb{F} ; f, g)$.

We have examples of Minkowski planes in types VII.F.23, VII.F.1, I.D.1, I.A.3 and I.A.1, see the following section 6 . We do not know at present whether or not types V.E.1, III.C.1 or I.B.1 do occur.

Remark 5.6. Theorems 3.2 and 5.3 do not involve (SA). Combining these Theorems and using Lemma 5.4 we readily find that the automorphism group $\Gamma_{f, g}$ of a Minkowski plane $\mathcal{M}(\mathbb{F} ; f, g)$ can be of type F.23, F.1, E.1, D.1, C.1, B.1, A.3 or A.1. Clearly, types F.23 and F.1 imply type VII and type E.1 implies type at least V, see Remark 4.3.2. Furthermore, type C.1 implies type at least III.

More precisely, one finds that $\Gamma_{f, g}$ is of type F.23, F.1, E.1, D.1, C.1, B.1, A.3 or A.1 if and only if $f$ and $g$ satisfy the same conditions as for type VII.F.23, VII.F.1, V.E.1, I.D.1, III.C.1, I.B.1, I.A.3 or I.A.1, respectively, in the Classification Theorem 5.5. No other types can occur among the Minkowski planes $\mathcal{M}(\mathbb{F} ; f, g)$. We therefore conjecture that Theorem 5.5 remains valid for any half-ordered field $\mathbb{F}$ even if (SA) is not satisfied for $\mathbb{F}$.

Remark 5.7. In [12] H.-J. Kroll and A. Matraś investigated Miquelian pairs of points in Minkowski planes. An inversion in a Minkowski plane $\mathcal{M}$ is an automorphism $\sigma$ of $\mathcal{M}$ that exchanges (+)- and (-)-parallel classes and has the property that $a, \sigma(a), b$ and $\sigma(b)$ are on a circle for any points $a$ and $b$ with $b$ parallel to neither 
$a$ nor $\sigma(a)$. We then say that a 2-set $\{p, q\}$ of points $p$ and $q$ in $\mathcal{M}$ is Miquelian if the collection of all inversions of $\mathcal{M}$ that interchange $p$ and $q$ operates transitively on each circle $C$ through $p$ and $q$ minus the two points $p$ and $q$. For a subgroup of the automorphism group of $\mathcal{M}$ H.-J. Kroll and A. Matraś determined the collection of all Miquelian pairs of points such that the corresponding inversions are in the subgroup. They obtained 9 types, cf. [12, Theorem 3.6], of which only five can occur as types for the full automorphism group. Following we list those types where $M$ denotes the set of all Miquelian unordered pairs of points in the Minkowski plane.

$M_{0} . M=\emptyset$;

$M_{1} . M=\{\{p, q\}\}$ for some nonparallel points $p$ and $q$;

$M_{2} . M=\left\{\{p, q\},\left\{|p|_{+} \cap|q|_{-},|p|_{-} \cap|q|_{+}\right\}\right\}$for some nonparallel points $p$ and $q$;

$M_{3} . M=\{\{p, q\} \mid p, q \in C, p \neq q\}$ for some circle $C$;

$M_{4} . M$ consists of all pairs of nonparallel points.

By [15, Theorem 1.3] the Miquelian Minkowski planes are the only planes of type $M_{4}$. Furthermore, if $\mathcal{M}$ is $(p, q)$-Miquelian, then $\mathcal{M}$ also is $(p, q)$-transitive, cf. [12, Lemma 2.2] and [15, (4.1.ii)]. In particular, type 1 implies type $M_{0}$. For our Minkowski planes $\mathcal{M}(\mathbb{F} ; f, g)$ the converse is also true. For example, if $\mu \in \mathrm{Mul}^{+}(\mathbb{F})$, then $\mathcal{M}(\mathbb{F} ; \mu, \mu)$ is $((\infty, \infty),(0,0))$ - and $((\infty, 0),(0, \infty))$-transitive. In this case each $\operatorname{map}(x, y) \mapsto(r / y, r / x)$ and $(x, y) \mapsto(r y, r x)$ for $r \in \mathbb{F}, r \neq 0$, is an inversion of $\mathcal{M}(\mathbb{F} ; \mu, \mu)$ that interchanges $(\infty, \infty)$ and $(0,0)$ and $(\infty, 0)$ and $(0, \infty)$, respectively. Hence, type 3 implies type $M_{2}$. In summary we obtain that the automorphism group $\Gamma_{f, g}$ of a Minkowski plane $\mathcal{M}(\mathbb{F} ; f, g)$ is of type $M_{4}, M_{2}$ or $M_{0}$ if and only if it is of Klein-Kroll type 23, 3 or 1 , respectively.

\section{SOME EXAMPLES}

In this section we specialise the Classification Theorem 5.5 to various half-ordered fields.

6.1 Finite fields. As seen in Example 4.1.2 every finite half-ordered field satisfies (SA). By [1] every order-preserving permutation of a finite half-ordered field $\mathbb{F}$ of odd order is an affine transformation of an automorphism of $\mathbb{F}$. Hence in this case every order-preserving permutation of $\overline{\mathrm{F}}$ belongs already to $\mathrm{PCL}_{2}^{+}$. Therefore the finite planes in our family are all of type VII.F. More precisely, a Minkowski plane $\mathcal{M}(\mathbb{F} ; f, g)$ is of type VII.F.23 if and only if $f \mathrm{PGL}_{2}^{+}=g \mathrm{PGL}_{2}^{+}$(Miquelian planes) and of type VII.F.1 if and only if $f \mathrm{PGL}_{2}^{+} \neq g \mathrm{PGL}_{2}^{+}$.

6.2 Subfields of $\mathbb{R}$ with the Euclidean ordering. By Example 4.1.1 every subfield $\mathbb{F}$ of $\mathbb{R}$ with the familiar Euclidean ordering satisfies $(\mathrm{SA})$ and $\mathrm{Aut}^{+}(\mathbb{F})$ is trivial. Hence $\mathrm{PCL}_{2}^{+}=\mathrm{PGL}_{2}^{+}$. Furthermore, $\mathbb{Q}$ is dense in $\mathbb{F}$ and every monotonic map is continuous. Hence it readily follows that $\operatorname{Add}^{+}(\mathbb{F})=\left\{\mu_{r} \mid r \in \mathbb{F}, r>0\right\}$ since every additive map is linear over $\mathbb{Q}$, cf. $[2,4.1 .3]$. Thus $\operatorname{Add}^{+}(\mathbb{F}) \subseteq \mathrm{PGL}_{2}^{+}$ and therefore $\Delta=\mathrm{PGL}_{2}^{+}$. 
From $\Delta=\mathrm{P}^{+} L_{2}^{+}=\mathrm{PGL}_{2}^{+}$we see that types VII.F.1, V.E.1, III.C.1 and I.B.1 are empty. In summary the automorphism group $\Gamma_{f, g}$ of a Minkowski plane $\mathcal{M}(\mathbb{F} ; f, g)$ where $\mathbb{F}$ is a subfield of $\mathbb{R}$ with the Euclidean ordering is of class

VII.F.23 if and only if $f, g \in \mathrm{PGL}_{2}^{+}$(Miquelian Minkowski planes);

I.D.1 if and only if $f \in \mathrm{PGL}_{2}^{+}, g \in \Pi^{+}(\overline{\mathbb{F}}) \backslash \mathrm{PGL}_{2}^{+}$or $f \in \Pi^{+}(\overline{\mathbb{F}}) \backslash \mathrm{PGL}_{2}^{+}$, $g \in \mathrm{PGL}_{2}^{+}$

I.A.3 if and only if $f, g \in \Phi \backslash \mathrm{PGL}_{2}^{+}$and $f$ and $g$ are in the same double coset of $\mathrm{PGL}_{2}^{+}$

I.A.1 if and only if $f, g \in \Pi^{+}(\overline{\mathbb{F}}) \backslash \mathrm{PGL}_{2}^{+}$and $f \in \Pi^{+}(\overline{\mathbb{F}}) \backslash \Phi$ or $g \in \Pi^{+}(\overline{\mathbb{F}}) \backslash \Phi$ or $f, g \in \Phi \backslash \mathrm{PGL}_{2}^{+}$but $f, g$ are in different double cosets of $\mathrm{PGL}_{2}^{+}$.

No other types occur.

Note that for $\mathbb{F}=\mathbb{R}$ the group $\mathrm{PGL}_{2}^{+}$becomes the projective special linear group $\operatorname{PSL}(2, \mathbb{R})$. This group was used in [17]. Furthermore all of the above four types do actually occur in this case. E.g. the map $\phi: x \mapsto x^{3}$ belongs to $\Phi(\mathbb{R}) \backslash \mathrm{PGL}^{+}(2, \mathbb{R})$ and with this map one obtains Minkowski planes of type I.D.1, I.A.3 and I.A.1. (For example, $\mathcal{M}(\mathbb{R} ; i d, \phi), \mathcal{M}(\mathbb{R} ; \phi, \phi), \mathcal{M}\left(\mathbb{R} ; \phi^{2}, \phi\right)$ are Minkowski planes of the respective types. Of course, $\mathcal{M}(\mathbb{R} ; i d, i d)$ is of type VII.F.23.) Note however, that not all of the above types may occur for a particular subfield $\mathbb{F}$ of $\mathbb{R}$; of course type VII.F.23 always occurs.

6.3 Subfields of the $p$-adic numbers $\mathbb{Q}_{p}$ with the $p$-adic ordering. Since $\mathbb{Q}$ is dense in $\mathbb{Q}_{p}$, one can follow the same steps as in 6.2. They result in exactly the same types VII.F.23, I.D.1, I.A.3 and I.A.1.

So far we have accounted for types VII.F.23, VII.F.1, I.D.1, I.A.3 and I.A.1. In order to find models of Minkowski planes whose automorphism groups are of one of the remaining types V.E.1, III.C.1 or I.B.1 one essentially has to find half-ordered fields $\mathbb{F}$ that satisfy (SA) and that admit permutations in $\Delta \backslash \mathrm{P}_{2}^{+}$and in $\Pi^{+}(\overline{\mathbb{F}}) \backslash \Delta$. This is not too difficult. However, given such permutations one still has to verify the fixed point property (FP). For example, the field $\mathbb{F}=\mathbb{Z}_{p}(X)$ of rational functions over $\mathbb{Z}_{p}$ (see 4.1.3) admits many permutations in $\Delta \backslash \mathrm{P}_{2}^{+}$, e.g., for $p>2$ let $\delta$ be defined by $\delta(h(X)+q(X))=-h(X)+q(X)$ where $h(X)$ is polynomial and $q(X)$ is a rational function such that the degree of the polynomial in the numerator is strictly less than the degree of the polynomial in the denominator. (By the division algorithm every rational function has an essentially unique representation in this form.) Then it readily follows that $\delta$ is an additive order-preserving permutation and that $\delta$ does not belong to $\mathrm{PLL}_{2}^{+}$. However, $i d$ and $\delta$ do not satisfy (FP). We therefore leave it open for now whether or not models of types V.E.1, III.C.1 or I.B.1 do exist.

\section{REFERENCES}

[1] Carlitz, L.: A theorem "on permutations in a finite field. Proc. Amer. Math. Soc. 11 (1960), 456-459 
[2] Dieudonné, J.: Foundations of Modern Analysis. Academic Press, New York, 1969

[3] JAKÓBOWSKI, J.: Conjugate points of a translation plane of order 9 and its extensibility to a Minkowski plane. Zeszyty Nauk. Geom. 17 (1988), $85-96$

[4] JАKÓBOWSKI, J.M.: A new generalization of Moulton affine planes. Geom. Dedicata 42 (1992), 243-253

[5] JAKÓBOWSKI, J.M.: A full generalization of Moulton's method applied to Minkowski axioms. Zeszyty Nauk. Geom. 20 (1993), 13-21

[6] JAKÓBOWsKI, J.M.: A new construction for Minkowski planes. Geom. Dedicata 69 (1998), 179-188

[7] KLEIN, M.: Classification of Minkowski planes by transitive groups of homotheties. J. Geom. 43 (1992), 116-128

[8] Klein, M. AND Kroll, H.-J.: A classification of Minkowski planes. J. Geom. 36 (1989), 99-109

[9] Klein, M. AND KROLL, H.-J.: On Minkowski planes with transitive groups of homotheties. Abh. Math. Sem. Univ. Hamburg 64 (1994), $303-313$

[10] KROLL, H.-J.: Eine Kennzeichnung der miquelschen Minkowski-Ebenen durch Transitivitätseigenschaften. Geom. Dedicata 58 (1995), 75-77

[11] Kroll, H.-J. AND MATraś, A.: Minkowski planes with miquelian pairs. Beitr. Algebra Geom. 38 (1997), 99-109

[12] Kroll, H.-J. AND MATrAŚ, A.: Minkowski planes admitting automorphism groups of small type. Preprint

[13] LANG, S.: Algebra. 7th ed., Addison-Wesley, Reading, Massachusetts 1977

[14] Meschiar, I.M. And Quattrocchi, P.: Una classificazione delle strutture di incidenza associate a insiemi di sostituzioni strettamente 3transitivi finiti. Atti Sem. Mat. Fis Univ. Modena 24 (1975), 123-141

[15] Perscy, N.: Les plans de Minkowski possedant des inversions sont miquellien. Simon Stevin 57 (1983), 15-35

[16] STEINkE, G.F.: A remark on Benz planes of order 9. Ars Comb. 34 (1992), 257-267

[17] STEINKE, G.F .: A family of 2-dimensional Minkowski planes with small automorphism groups. Result. Math. 26 (1994), 131-142

[18] STEINKE, G.F.: Topological circle geometries. in: Handbook of Incidence Geometry (ed. F. Buekenhout) Elsevier 1995, pp. 1327-1354

[19] STEINKE, G.F.: Semiclassical projective planes over half-ordered fields. Geom. Dedicata 58 (1995), 21-44

[20] STEINKE, G.F.: A family of Minkowski planes over half-ordered fields. Beitr. Algebra Geom. 37 (1996), 355-366 